\title{
Alkali Metal Salts with Designable Aryltrifluoroborate Anions
}

Kazuki Iwasaki, ${ }^{\dagger}$ Kazuki Yoshii, ${ }^{\dagger}$ Seiji Tsuzuki, ${ }^{\dagger}$ Hajime Matsumoto, ${ }^{\dagger, \S}$ Tetsuya Tsuda $^{*}{ }^{\dagger}$, and Susumu Kuwabata ${ }^{*} \dagger, \S$

†Department of Applied Chemistry, Graduate School of Engineering, Osaka University, 2-1 Yamada-oka, Suita, Osaka 565-0871, Japan

${ }^{\ddagger}$ Research Center for Computational Design of Advanced Functional Materials, National Institute of Advanced Industrial Science and Technology (AIST), 1-1-1 Umezono, Tsukuba, Ibaraki 305-8568, Japan

${ }^{\S}$ Department of Energy and Environment, Research Institute of Electrochemical Energy, National Institute of Advanced Industrial Science and Technology (AIST), 1-8-31 Midorigaoka, Ikeda, Osaka 563-8577, Japan

\section{Supporting Information}

\section{Characterization of alkali metal salts with aryltrifluoroborate}

${ }^{1} \mathrm{H},{ }^{13} \mathrm{C},{ }^{19} \mathrm{~F}$, and ${ }^{11} \mathrm{~B}$ NMR spectra were recorded on a JEOL JNM-ECS400 spectrometer operating at 400, 100, 376, and $128 \mathrm{MHz}$, respectively. $\mathrm{CD}_{3} \mathrm{CN}$ and DMSO- $d^{6}$ were used as the solvent for the samples in this study. ${ }^{1} \mathrm{H}$ and ${ }^{13} \mathrm{C}$ NMR spectra were referenced to the residual solvent signal as the internal standard: $\mathrm{CD}_{3} \mathrm{CN} \delta=1.93 \mathrm{ppm}\left({ }^{1} \mathrm{H}\right)$ and $\delta=1.3 \mathrm{ppm}\left({ }^{13} \mathrm{C}\right)$; DMSO- $d^{6} \delta=2.5 \mathrm{ppm}\left({ }^{1} \mathrm{H}\right)$ and $\delta=39.5 \mathrm{ppm}\left({ }^{13} \mathrm{C}\right)$. Chemical shift values in ${ }^{19} \mathrm{~F}$ and ${ }^{11} \mathrm{~B}$ NMR were reported relative to the external references of $\mathrm{C}_{6} \mathrm{H}_{5} \mathrm{CF}_{3} \delta=-63.7 \mathrm{ppm}\left({ }^{19} \mathrm{~F}\right)$ and $\mathrm{BF}_{3} \cdot \mathrm{OEt}_{2} \delta=0.0 \mathrm{ppm}\left({ }^{11} \mathrm{~B}\right)$. High-resolution mass spectrometry (HRMS) were operated on a JEOL JMS-700 MStation mass spectrometer using fast atom bombardment (FAB) ionization. HRMS and Elemental analysis $(\mathrm{C}, \mathrm{H}$, and $\mathrm{N})$ were performed at Instrumental Analysis Center, Faculty of Engineering, Osaka University.

Potassium phenyltrifluoroborate $\left.\left(\mathbf{K}_{\mathbf{P h B F}}\right]\right)$ : yield: $90 \%$, white solid. ${ }^{1} \mathrm{H} \mathrm{NMR}(400 \mathrm{MHz}$, $\left.\mathrm{CD}_{3} \mathrm{CN}\right): \sigma 7.07\left(\mathrm{~m}, 1 \mathrm{H}\right.$, arom. H), $7.14\left(\mathrm{t}, 2 \mathrm{H}, J=7.2 \mathrm{~Hz}\right.$, arom. H), $7.40\left(\mathrm{~d}, 2 \mathrm{H},{ }^{3} J_{\mathrm{HH}}=7.2 \mathrm{~Hz}\right.$, arom. H). ${ }^{13} \mathrm{C}$ NMR (100 MHz, $\left.\mathrm{CD}_{3} \mathrm{CN}\right): \sigma 126.5$ (s), 127.6 (s), 132.3 (s). ${ }^{19} \mathrm{~F}$ NMR (376 MHz, $\left.\mathrm{CD}_{3} \mathrm{CN}\right): \sigma-141.6\left(\mathrm{q}, 3 \mathrm{~F},{ }^{1} J_{\mathrm{BF}}=51.7 \mathrm{~Hz}, \mathrm{~B} F_{3}\right) .{ }^{11} \mathrm{~B} \mathrm{NMR}\left(128 \mathrm{MHz}, \mathrm{CD}_{3} \mathrm{CN}\right): \sigma 3.84\left(\mathrm{q},{ }^{1} J_{\mathrm{BF}}=56.4\right.$ $\mathrm{Hz}$ ). HRMS (FAB), $m / z$ : calcd. for $\mathrm{C}_{6} \mathrm{H}_{5} \mathrm{BF}_{3} \mathrm{~K}_{2}{ }^{+} 222.9705[\mathrm{M}+\mathrm{K}]^{+}$; found 222.9709. Elemental analysis calcd. for $\mathrm{C}_{6} \mathrm{H}_{5} \mathrm{BF}_{3} \mathrm{~K}$ : C, 39.16; H, 2.74; found: C, 39.19; H, 2.97.

Potassium (meta-methylphenyl)trifluoroborate $\left(\mathbf{K}\left[\mathbf{m}-\mathbf{M e C}_{\mathbf{6}} \mathbf{H}_{\mathbf{4}} \mathbf{B F}_{3}\right]\right)$ : yield: $97 \%$, white solid. ${ }^{1} \mathrm{H}$ NMR (400 MHz, $\left.\mathrm{CD}_{3} \mathrm{CN}\right): \sigma 2.25\left(\mathrm{~s}, 3 \mathrm{H}, \mathrm{CH}_{3}\right), 6.92(\mathrm{t}, 1 \mathrm{H}, J=8.8 \mathrm{~Hz}$, arom. H), $7.04(\mathrm{q}, 1 \mathrm{H}, J=$ $7.8 \mathrm{~Hz}$, arom. H), 7.18-7.27 (m, 2H, arom. H). $\left.{ }^{13} \mathrm{C} \mathrm{NMR} \mathrm{(100} \mathrm{MHz,} \mathrm{CD}_{3} \mathrm{CN}\right): \sigma 21.7\left(\mathrm{~s}, \mathrm{CH}_{3}\right), 127.3$ 
(d, $J=23.8 \mathrm{~Hz}), 127.6(\mathrm{~d}, J=9.5 \mathrm{~Hz}), 129.3(\mathrm{~s}), 133.1(\mathrm{~s}), 136.5(\mathrm{~d}, J=11.6 \mathrm{~Hz}) .{ }^{19} \mathrm{~F}$ NMR $(376$ $\left.\mathrm{MHz}, \mathrm{CD}_{3} \mathrm{CN}\right): \sigma-140.7\left(\mathrm{~d}, 3 \mathrm{~F},{ }^{1} J_{\mathrm{BF}}=62.4 \mathrm{~Hz}, \mathrm{~B} F_{3}\right) .{ }^{11} \mathrm{~B} \mathrm{NMR}\left(128 \mathrm{MHz}, \mathrm{CD}_{3} \mathrm{CN}\right): \sigma 3.91\left(\mathrm{~d},{ }^{1} J_{\mathrm{BF}}\right.$ $=56.3 \mathrm{~Hz}$ ). HRMS (FAB), $m / z$ : calcd. for $\mathrm{C}_{7} \mathrm{H}_{7} \mathrm{BF}_{3} \mathrm{~K}_{2}^{+} 236.9862[\mathrm{M}+\mathrm{K}]^{+}$; found 236.9869 . Elemental analysis calcd. for $\mathrm{C}_{7} \mathrm{H}_{7} \mathrm{BF}_{3} \mathrm{~K}$ : $\mathrm{C}, 42.46$; $\mathrm{H}, 3.56$; found: $\mathrm{C}, 42.17$; $\mathrm{H}, 3.72$.

Potassium (meta-tert-butylphenyl)trifluoroborate $\left(\mathbf{K}\left[m-{ }^{t} \mathbf{B u C}_{6} \mathbf{H}_{4} \mathbf{B F}_{3}\right]\right)$ : yield: $99 \%$, white solid. ${ }^{1} \mathrm{H}$ NMR (400 MHz, $\left.\mathrm{CD}_{3} \mathrm{CN}\right): \sigma 1.27\left(\mathrm{~s}, 9 \mathrm{H}, \mathrm{C}\left(\mathrm{CH}_{3}\right)_{3}\right), 7.06-7.23(\mathrm{~m}, 3 \mathrm{H}$, arom. H), 7.49 (s, 1H, arom. H). ${ }^{13} \mathrm{C} \mathrm{NMR} \mathrm{(100} \mathrm{MHz,} \mathrm{CD}_{3} \mathrm{CN}$ ): $\sigma 31.9$ (s), 35.0 (s), 123.5 (s), 127.3 (s), 128.9 (s), 129.5 (s), 149.6 (s). ${ }^{19} \mathrm{~F}$ NMR $\left(376 \mathrm{MHz}, \mathrm{CD}_{3} \mathrm{CN}\right): \sigma-142.4\left(\mathrm{~d}, 3 \mathrm{~F},{ }^{1} J_{\mathrm{BF}}=57.5 \mathrm{~Hz}, \mathrm{~B} F_{3}\right) .{ }^{11} \mathrm{~B} \mathrm{NMR}(128 \mathrm{MHz}$, $\left.\mathrm{CD}_{3} \mathrm{CN}\right): \sigma 4.03\left(\mathrm{~d},{ }^{1} J_{\mathrm{BF}}=55.0 \mathrm{~Hz}\right)$. HRMS (FAB), $m / z$ : calcd. for $\mathrm{C}_{10} \mathrm{H}_{13} \mathrm{BF}_{3} \mathrm{~K}_{2}{ }^{+} 279.0331[\mathrm{M}+$ $\mathrm{K}]^{+}$; found 279.0343. Elemental analysis calcd. for $\mathrm{C}_{10} \mathrm{H}_{13} \mathrm{BF}_{3} \mathrm{~K}$ : C, 50.02; H, 5.46; found: C, 49.70; $\mathrm{H}, 5.55$.

Potassium (meta-trifluoromethylphenyl)trifluoroborate $\left(\mathbf{K}\left[m-\mathbf{C F}_{3} \mathbf{C}_{6} \mathbf{H}_{\mathbf{4}} \mathbf{B F}_{3}\right]\right)$ : yield: $93 \%$, white solid. ${ }^{1} \mathrm{H}$ NMR (400 MHz, $\left.\mathrm{CD}_{3} \mathrm{CN}\right): \sigma 7.33\left(\mathrm{t}, 1 \mathrm{H}, J=7.6 \mathrm{~Hz}\right.$, arom. H), $7.41\left(\mathrm{~d}, 1 \mathrm{H},{ }^{3} J_{\mathrm{HH}}=7.6 \mathrm{~Hz}\right.$, arom. H), 7.67-7.71 (m, 2H, arom. H). $\left.{ }^{13} \mathrm{C} \mathrm{NMR} \mathrm{(100} \mathrm{MHz,} \mathrm{CD}_{3} \mathrm{CN}\right): \sigma 123.2\left(\mathrm{q},{ }^{3} J_{\mathrm{CF}}=3.8 \mathrm{~Hz}\right.$ ), 126.4 (q, $\left.{ }^{1} J_{\mathrm{CF}}=272.6 \mathrm{~Hz}, C \mathrm{~F}_{3}\right), 128.1$ (s), 128.5 (s), 129.0 (q, $\left.{ }^{2} J_{\mathrm{CF}}=30.6 \mathrm{~Hz}\right), 136.1$ (s). ${ }^{19} \mathrm{~F} \mathrm{NMR}$ $\left(376 \mathrm{MHz}, \mathrm{CD}_{3} \mathrm{CN}\right): \sigma-143.6\left(\mathrm{~d}, 3 \mathrm{~F},{ }^{1} J_{\mathrm{BF}}=57.5 \mathrm{~Hz}, \mathrm{~B} F_{3}\right),-63.1\left(\mathrm{~s}, 3 \mathrm{~F}, \mathrm{CF}_{3}\right) .{ }^{11} \mathrm{~B} \mathrm{NMR}(128 \mathrm{MHz}$, $\mathrm{CD}_{3} \mathrm{CN}$ ): $\sigma 3.51\left(\mathrm{q},{ }^{1} J_{\mathrm{BF}}=50.1 \mathrm{~Hz}\right.$ ). HRMS (FAB), $m / z$ : calcd. for $\mathrm{C}_{7} \mathrm{H}_{4} \mathrm{BF}_{6} \mathrm{~K}_{2}{ }^{+} 290.9579[\mathrm{M}+\mathrm{K}]^{+}$; found 290.9579. Elemental analysis calcd. for $\mathrm{C}_{7} \mathrm{H}_{4} \mathrm{BF}_{6} \mathrm{~K}$ : C, 33.36; H, 1.60; found: C, 33.48; $\mathrm{H}$, 1.93.

Potassium (meta-fluorophenyl)trifluoroborate $\left(\mathbf{K}\left[\boldsymbol{m}-\mathbf{F C}_{\mathbf{6}} \mathbf{H}_{\mathbf{4}} \mathbf{B F}_{3}\right]\right)$ : yield: $82 \%$, white solid. ${ }^{1} \mathrm{H}$ NMR (400 MHz, $\mathrm{CD}_{3} \mathrm{CN}$ ): $\sigma 6.79\left(\mathrm{t}, 1 \mathrm{H}, J=8.9 \mathrm{~Hz}\right.$, arom. H), $7.09\left(\mathrm{~d}, 1 \mathrm{H},{ }^{3} J_{\mathrm{HH}}=10.0 \mathrm{~Hz}\right.$, arom. $\mathrm{H}), 7.12-7.21\left(\mathrm{~m}, 2 \mathrm{H}\right.$, arom. H). ${ }^{13} \mathrm{C} \mathrm{NMR}\left(100 \mathrm{MHz}, \mathrm{CD}_{3} \mathrm{CN}\right): \sigma 113.0\left(\mathrm{~d},{ }^{2} J_{\mathrm{CF}}=21.1 \mathrm{~Hz}\right), 118.1(\mathrm{~d}$, $\left.{ }^{2} J_{\mathrm{CF}}=17.3 \mathrm{~Hz}\right), 128.0(\mathrm{~s}), 129.5(\mathrm{~s}), 163.5\left(\mathrm{~d},{ }^{1} J_{\mathrm{CF}}=233.3 \mathrm{~Hz}\right) \cdot{ }^{19} \mathrm{~F}$ NMR $\left(376 \mathrm{MHz}, \mathrm{CD}_{3} \mathrm{CN}\right): \sigma$ $-143.3\left(\mathrm{q}, 3 \mathrm{~F},{ }^{1} J_{\mathrm{BF}}=51.7 \mathrm{~Hz}, \mathrm{~B} F_{3}\right),-117.8(\mathrm{~s}, 1 \mathrm{~F}, 3-F) .{ }^{11} \mathrm{~B} \mathrm{NMR}\left(128 \mathrm{MHz}, \mathrm{CD}_{3} \mathrm{CN}\right): \sigma 3.35$ (q, ${ }^{1} J_{\mathrm{BF}}=53.3 \mathrm{~Hz}$ ). HRMS (FAB), $m / z$ : calcd. for $\mathrm{C}_{6} \mathrm{H}_{4} \mathrm{BF}_{4} \mathrm{~K}_{2}{ }^{+} 240.9611[\mathrm{M}+\mathrm{K}]^{+}$; found 240.9627 . Elemental analysis calcd. for $\mathrm{C}_{6} \mathrm{H}_{4} \mathrm{BF}_{4} \mathrm{~K}$ : C, 35.68; H, 2.00; found: C, 35.66; H, 2.06.

Potassium (meta-trifluoromethoxyphenyl)trifluoroborate $\left(\mathrm{K}\left[m-\mathrm{OCF}_{\mathbf{3}} \mathrm{C}_{\mathbf{6}} \mathrm{H}_{\mathbf{4}} \mathrm{BF}_{\mathbf{3}}\right]\right)$ : yield: $52 \%$, pale yellow solid. ${ }^{1} \mathrm{H}$ NMR (400 MHz, $\left.\mathrm{CD}_{3} \mathrm{CN}\right): \sigma 7.02\left(\mathrm{~d}, 1 \mathrm{H},{ }^{3} J_{\mathrm{HH}}=8.2 \mathrm{~Hz}\right.$, arom. H), $7.25(\mathrm{t}, 1 \mathrm{H}$, $J=7.8 \mathrm{~Hz}$, arom. H), 7.30 (s, 1H, arom. H), 7.42 (d, $1 \mathrm{H},{ }^{3} J_{\mathrm{HH}}=7.3 \mathrm{~Hz}$, arom. H). ${ }^{13} \mathrm{C}$ NMR (100 $\mathrm{MHz}, \mathrm{CD}_{3} \mathrm{CN}$ ): $\sigma 119.2$ (s), 121.4 (q, $\left.{ }^{1} J_{\mathrm{CF}}=235.7 \mathrm{~Hz}, \mathrm{OCF}_{3}\right), 124.2$ (s), $129.4(\mathrm{~s}), 131.1$ (s), 149.5 (s). ${ }^{19} \mathrm{~F}$ NMR $\left(376 \mathrm{MHz}, \mathrm{CD}_{3} \mathrm{CN}\right): \sigma-143.4\left(\mathrm{~d}, 3 \mathrm{~F},{ }^{1} J_{\mathrm{BF}}=58.4 \mathrm{~Hz}, \mathrm{~B} F_{3}\right),-58.6\left(\mathrm{~s}, 3 \mathrm{~F}, \mathrm{OC} F_{3}\right) .{ }^{11} \mathrm{~B}$ NMR (128 MHz, $\mathrm{CD}_{3} \mathrm{CN}$ ): $\sigma 3.47$ (q, ${ }^{1} J_{\mathrm{BF}}=48.1 \mathrm{~Hz}$ ). HRMS (FAB), $m / z$ : calcd. for $\mathrm{C}_{7} \mathrm{H}_{4} \mathrm{OBF}_{6} \mathrm{~K}_{2}{ }^{+}$ $306.9528[\mathrm{M}+\mathrm{K}]^{+}$; found 306.9533. Elemental analysis calcd. for $\mathrm{C}_{7} \mathrm{H}_{4} \mathrm{OBF} \mathrm{K}_{6}$ : C, 31.37; $\mathrm{H}, 1.50$; found: C, 30.70; H, 1.86 . 
Potassium (meta-methylthiophenyl)trifluoroborate $\left(\mathrm{K}\left[m-\mathrm{SMeC}_{6} \mathbf{H}_{4} \mathrm{BF}_{3}\right]\right)$ : yield: $72 \%$, pale yellow solid. ${ }^{1} \mathrm{H}$ NMR (400 MHz, $\left.\mathrm{CD}_{3} \mathrm{CN}\right): \sigma 2.41\left(\mathrm{~s}, 3 \mathrm{H}, \mathrm{SCH}_{3}\right), 7.04\left(\mathrm{~d}, 1 \mathrm{H},{ }^{3} \mathrm{~J}_{\mathrm{HH}}=7.8 \mathrm{~Hz}\right.$, arom. H), 7.11 (t, $1 \mathrm{H}, J=7.6 \mathrm{~Hz}$, arom. H), 7.22 (d, 1H, ${ }^{3} J_{\mathrm{HH}}=7.3 \mathrm{~Hz}$, arom. H), 7.36 (s, 1H, arom. H). ${ }^{13} \mathrm{C}$ NMR (100 MHz, $\left.\mathrm{CD}_{3} \mathrm{CN}\right): \sigma 16.0$ (s, $\mathrm{SCH}_{3}$ ), 125.1 (s), 128.4 (s), 129.3 (s), 130.7 (s), 136.9 (s). ${ }^{19} \mathrm{~F}$ NMR $\left(376 \mathrm{MHz}, \mathrm{CD}_{3} \mathrm{CN}\right): \sigma-142.9\left(\mathrm{~d}, 3 \mathrm{~F},{ }^{1} J_{\mathrm{BF}}=49.7 \mathrm{~Hz}, \mathrm{~B} F_{3}\right) .{ }^{11} \mathrm{~B}$ NMR $\left(128 \mathrm{MHz}, \mathrm{CD}_{3} \mathrm{CN}\right)$ : $\sigma 3.76\left(\mathrm{~d},{ }^{1} J_{\mathrm{BF}}=44.9 \mathrm{~Hz}\right)$. HRMS (FAB), $m / z$ : calcd. for $\mathrm{C}_{7} \mathrm{H}_{7} \mathrm{SBF}_{3} \mathrm{~K}_{2}{ }^{+} 268.9582[\mathrm{M}+\mathrm{K}]^{+}$; found 268.9591. Elemental analysis calcd. for $\mathrm{C}_{7} \mathrm{H}_{7} \mathrm{SBF}_{3} \mathrm{~K}$ : C, 36.54; H, 3.07; found: C, 35.93; H, 3.21 .

Potassium (meta-methylsulfonylphenyl)trifluoroborate $\left(\mathrm{K}\left[m-\mathrm{SO}_{2} \mathrm{MeC}_{6} \mathbf{H}_{4} \mathrm{BF}_{3}\right]\right)$ : yield: $86 \%$, white solid. ${ }^{1} \mathrm{H}$ NMR (400 MHz, $\mathrm{CD}_{3} \mathrm{CN}$ ): $\sigma 2.98$ (s, 3H, $\mathrm{SO}_{2} \mathrm{CH}_{3}$ ), 7.38 (t, $1 \mathrm{H}, J=7.8 \mathrm{~Hz}$, arom. H), $7.64\left(\mathrm{~d}, 1 \mathrm{H},{ }^{3} J_{\mathrm{HH}}=7.7 \mathrm{~Hz}\right.$, arom. H), $7.74\left(\mathrm{~d}, 1 \mathrm{H},{ }^{3} J_{\mathrm{HH}}=7.3 \mathrm{~Hz}\right.$, arom. H), $7.92(\mathrm{~s}, 1 \mathrm{H}$, arom. H). ${ }^{13} \mathrm{C}$ NMR (100 MHz, $\mathrm{CD}_{3} \mathrm{CN}$ ): $\sigma 44.6$ (s, $\mathrm{SO}_{2} \mathrm{CH}_{3}$ ), 125.2 (s), 128.5 (s), 130.4 (s), 137.6 (s), 140.0 (s). ${ }^{19} \mathrm{~F}$ NMR $\left(376 \mathrm{MHz}, \mathrm{CD}_{3} \mathrm{CN}\right): \sigma-143.6\left(\mathrm{q}, 3 \mathrm{~F},{ }^{1} J_{\mathrm{BF}}=52.6 \mathrm{~Hz}, \mathrm{~B} F_{3}\right) .{ }^{11} \mathrm{~B} \mathrm{NMR}(128 \mathrm{MHz}$, $\left.\mathrm{CD}_{3} \mathrm{CN}\right): \sigma 3.31\left(\mathrm{~d},{ }^{1} J_{\mathrm{BF}}=50.8 \mathrm{~Hz}\right.$ ). HRMS (FAB), $m / z$ : calcd. for $\mathrm{C}_{7} \mathrm{H}_{7} \mathrm{O}_{2} \mathrm{SBF}_{3} \mathrm{~K}_{2}{ }^{+} 300.9481[\mathrm{M}+$ $\mathrm{K}]^{+}$; found 300.9492. Elemental analysis calcd. for $\mathrm{C}_{7} \mathrm{H}_{7} \mathrm{O}_{2} \mathrm{SBF}_{3} \mathrm{~K}: \mathrm{C}, 32.08$; H, 2.69; found: $\mathrm{C}$, $32.08 ; \mathrm{H}, 2.87$.

Potassium (meta-bromomethylphenyl)trifluoroborate $\left(\mathrm{K}\left[m-\mathrm{CH}_{2} \mathrm{BrC}_{6} \mathrm{H}_{4} \mathrm{BF}_{3}\right]\right)$ : yield: $81 \%$, white solid. ${ }^{1} \mathrm{H}$ NMR (400 MHz, $\mathrm{CD}_{3} \mathrm{CN}$ ): $\sigma 4.64$ (s, 2H, $\mathrm{CH}_{2} \mathrm{Br}$ ), 7.06-7.13 (m, 2H, arom. H), 7.26 $\left(\mathrm{d}, 1 \mathrm{H},{ }^{3} J_{\mathrm{HH}}=6.4 \mathrm{~Hz}\right.$, arom. H), $7.38\left(\mathrm{~s}, 1 \mathrm{H}\right.$, arom. H). ${ }^{13} \mathrm{C}$ NMR $\left(100 \mathrm{MHz}\right.$, DMSO- $\left.d^{6}\right): \sigma 36.6(\mathrm{~s}$, $\mathrm{CH}_{2} \mathrm{Br}$ ), 126.1 (s), 126.6 (s), 131.5 (s), 132.5 (s), 135.3 (s). ${ }^{19} \mathrm{~F}$ NMR (376 MHz, DMSO- $d^{6}$ ): $\sigma$ -141.7 (br, 3F, B $\left.F_{3}\right) .{ }^{11} \mathrm{~B}$ NMR (128 MHz, DMSO- $\left.d^{6}\right): \sigma 3.66$ (br). HRMS (FAB), $m / z$ : calcd. for $\mathrm{C}_{7} \mathrm{H}_{6} \mathrm{BrBF}_{3} \mathrm{~K}_{2}^{+} 314.8967[\mathrm{M}+\mathrm{K}]^{+}$; found 314.8979 .

Potassium (meta-cyanophenyl)trifluoroborate $\left(\mathbf{K}\left[\boldsymbol{m}-\mathbf{C N C}_{6} \mathbf{H}_{4} \mathbf{B F}_{3}\right]\right)$ : yield: $99 \%$, white solid. ${ }^{1} \mathrm{H}$ NMR (400 MHz, $\left.\mathrm{CD}_{3} \mathrm{CN}\right): \sigma 7.29\left(\mathrm{t}, 1 \mathrm{H}, J=11.5 \mathrm{~Hz}\right.$, arom. H), $7.44\left(\mathrm{dt}, 1 \mathrm{H}, J=7.6 \mathrm{~Hz},{ }^{4} J=1.6 \mathrm{~Hz}\right.$, arom. H), $7.69\left(\mathrm{~d}, 1 \mathrm{H},{ }^{3} J_{\mathrm{HH}}=7.3 \mathrm{~Hz}\right.$, arom. H), $7.72\left(\mathrm{~s}, 1 \mathrm{H}\right.$, arom. H). ${ }^{13} \mathrm{C} \mathrm{NMR} \mathrm{(100} \mathrm{MHz,}$ $\mathrm{CD}_{3} \mathrm{CN}$ ): $\sigma 111.2$ (s), 121.2 (s), 128.4 (s), 130.2 (s), 135.9 (s), 136.9 (s). ${ }^{19} \mathrm{~F}$ NMR (376 MHz, $\left.\mathrm{CD}_{3} \mathrm{CN}\right): \sigma-143.8\left(\mathrm{q}, 3 \mathrm{~F},{ }^{1} J_{\mathrm{BF}}=48.7 \mathrm{~Hz}, \mathrm{~B} F_{3}\right) .{ }^{11} \mathrm{~B} \mathrm{NMR}\left(128 \mathrm{MHz}, \mathrm{CD}_{3} \mathrm{CN}\right): \sigma 3.16\left(\mathrm{q},{ }^{1} J_{\mathrm{BF}}=49.4\right.$ $\mathrm{Hz}$ ). HRMS (FAB), $m / z$ : calcd. for $\mathrm{C}_{7} \mathrm{H}_{4} \mathrm{NBF}_{3} \mathrm{~K}_{2}{ }^{+} 247.9658[\mathrm{M}+\mathrm{K}]^{+}$; found 247.9661. Elemental analysis calcd. for $\mathrm{C}_{7} \mathrm{H}_{4} \mathrm{NBF}_{3} \mathrm{~K}$ : C, 40.22; H, 1.93; N, 6.70; found: $\mathrm{C}, 38.94 ; \mathrm{H}, 2.16 ; \mathrm{N}, 6.60$.

Potassium (meta-nitrophenyl)trifluoroborate $\left(\mathbf{K}\left[m-\mathrm{NO}_{2} \mathbf{C}_{6} \mathbf{H}_{4} \mathbf{B F}_{3}\right]\right)$ : yield: $85 \%$, yellow solid. ${ }^{1} \mathrm{H}$ NMR (400 MHz, $\mathrm{CD}_{3} \mathrm{CN}$ ): $\sigma 7.37$ (t, $1 \mathrm{H}, J=7.8 \mathrm{~Hz}$, arom. H), 7.80 (d, $1 \mathrm{H},{ }^{3} J_{\mathrm{HH}}=7.2 \mathrm{~Hz}$, arom. H), $7.94\left(\mathrm{~d}, 1 \mathrm{H},{ }^{3} J_{\mathrm{HH}}=8.0 \mathrm{~Hz}\right.$, arom. H), $8.21\left(\mathrm{~s}, 1 \mathrm{H}\right.$, arom. H). ${ }^{13} \mathrm{C}$ NMR $\left(100 \mathrm{MHz}, \mathrm{CD}_{3} \mathrm{CN}\right): \sigma 121.6$ (s), 126.4 (s), 128.7 (s), 138.9 (s), 148.6 (s). ${ }^{19} \mathrm{~F}$ NMR $\left(376 \mathrm{MHz}, \mathrm{CD}_{3} \mathrm{CN}\right): \sigma-143.8$ (q, $3 \mathrm{~F},{ }^{1} J_{\mathrm{BF}}=$ $\left.48.7 \mathrm{~Hz}, \mathrm{~B} F_{3}\right) .{ }^{11} \mathrm{~B}$ NMR $\left(128 \mathrm{MHz}, \mathrm{CD}_{3} \mathrm{CN}\right): \sigma 3.21\left(\mathrm{q},{ }^{1} J_{\mathrm{BF}}=50.8 \mathrm{~Hz}\right)$. HRMS (FAB), $m / z$ : calcd. 
for $\mathrm{C}_{6} \mathrm{H}_{4} \mathrm{NO}_{2} \mathrm{BF}_{3} \mathrm{~K}_{2}^{+}$267.9556 $[\mathrm{M}+\mathrm{K}]^{+}$; found 267.9566. Elemental analysis calcd. for $\mathrm{C}_{6} \mathrm{H}_{4} \mathrm{NO}_{2} \mathrm{BF}_{3} \mathrm{~K}: \mathrm{C}, 31.47 ; \mathrm{H}, 1.76 ; \mathrm{N}, 6.12$; found: $\mathrm{C}, 31.15 ; \mathrm{H}, 1.88 ; \mathrm{N}, 6.14$.

Potassium (meta-ethoxycarbonylphenyl)trifluoroborate $\left(\mathrm{K}\left[m-\mathrm{CO}_{2} \mathrm{EtC}_{6} \mathbf{H}_{4} \mathrm{BF}_{3}\right]\right)$ : yield: $97 \%$, white solid. ${ }^{1} \mathrm{H}$ NMR (400 MHz, $\mathrm{CD}_{3} \mathrm{CN}$ ): $\sigma 1.32$ (t, 3H, ${ }^{3} J_{\mathrm{HH}}=7.1 \mathrm{~Hz}, \mathrm{CO}_{2} \mathrm{CH}_{2} \mathrm{CH}_{3}$ ), 4.27 (q, 2H, $\left.{ }^{3} J_{\mathrm{HH}}=7.0 \mathrm{~Hz}, \mathrm{CO}_{2} \mathrm{CH}_{2} \mathrm{CH}_{3}\right), 7.25\left(\mathrm{t}, 1 \mathrm{H}, J=7.6 \mathrm{~Hz}\right.$, arom. H), $7.64\left(\mathrm{~d}, 1 \mathrm{H},{ }^{3} J_{\mathrm{HH}}=7.3 \mathrm{~Hz}\right.$, arom. H), $7.73\left(\mathrm{~d}, 1 \mathrm{H},{ }^{3} J_{\mathrm{HH}}=7.8 \mathrm{~Hz}\right.$, arom. H), 8.04 (s, 1H, arom. H). ${ }^{13} \mathrm{C}$ NMR $\left(100 \mathrm{MHz}, \mathrm{CD}_{3} \mathrm{CN}\right): \sigma 14.6$ (s), 61.2 (s), 127.5 (s), 127.7 (s), 129.6 (s), 133.2 (s), 137.1 (s), 168.5 (s). ${ }^{19} \mathrm{~F}$ NMR (376 MHz, $\left.\mathrm{CD}_{3} \mathrm{CN}\right): \sigma-143.2\left(\mathrm{~d}, 3 \mathrm{~F},{ }^{1} J_{\mathrm{BF}}=49.8 \mathrm{~Hz}, \mathrm{~B} F_{3}\right) .{ }^{11} \mathrm{~B} \mathrm{NMR}\left(128 \mathrm{MHz}, \mathrm{CD}_{3} \mathrm{CN}\right): \sigma 3.54\left(\mathrm{~d},{ }^{1} J_{\mathrm{BF}}=52.8\right.$ $\mathrm{Hz}$ ). HRMS (FAB), $m / z$ : calcd. for $\mathrm{C}_{9} \mathrm{H}_{9} \mathrm{O}_{2} \mathrm{BF}_{3} \mathrm{~K}_{2}{ }^{+} 294.9916[\mathrm{M}+\mathrm{K}]^{+}$; found 294.9916. Elemental analysis calcd. for $\mathrm{C}_{9} \mathrm{H}_{9} \mathrm{O}_{2} \mathrm{BF}_{3} \mathrm{~K}$ : C, 42.21; H, 3.54; found: $\mathrm{C}, 41.38$; H, 3.60.

Potassium (meta-carbamoylphenyl)trifluoroborate $\left(\mathrm{K}\left[m-\mathrm{CONH}_{2} \mathrm{C}_{6} \mathrm{H}_{4} \mathrm{BF}_{3}\right]\right)$ : yield: $70 \%$, white solid. ${ }^{1} \mathrm{H}$ NMR (400 MHz, DMSO- $\left.d^{6}\right): \sigma 7.00\left(\mathrm{~s}, 1 \mathrm{H}, \mathrm{CONH} H_{2}\right), 7.14$ (t, $1 \mathrm{H}, J=7.6 \mathrm{~Hz}$, arom. H), $7.45\left(\mathrm{~d}, 1 \mathrm{H},{ }^{3} J_{\mathrm{HH}}=7.3 \mathrm{~Hz}\right.$, arom. H), $7.54\left(\mathrm{~d}, 1 \mathrm{H},{ }^{3} J_{\mathrm{HH}}=7.8 \mathrm{~Hz}\right.$, arom. H), $7.76\left(\mathrm{~s}, 1 \mathrm{H}, \mathrm{CONH}{ }_{2}\right)$, 7.85 (s, 1H, arom. H). ${ }^{13} \mathrm{C}$ NMR (100 MHz, DMSO- $d^{6}$ ): $\sigma 124.4$ (s), 125.9 (s), 130.5 (s), 132.2 (s), 134.3 (s), 169.5 (s, $\left.\mathrm{CONH}_{2}\right) .{ }^{19} \mathrm{~F}$ NMR (376 MHz, DMSO- $\left.d^{6}\right): \sigma-141.5$ (br, 3F, B $\left.F_{3}\right) .{ }^{11} \mathrm{~B}$ NMR $(128$ MHz, DMSO- $d^{6}$ ): $\sigma 3.77$ (br). HRMS (FAB), $m / z$ : calcd. for $\mathrm{C}_{7} \mathrm{H}_{6} \mathrm{NOBF}_{3} \mathrm{~K}_{2}{ }^{+} 265.9763[\mathrm{M}+\mathrm{K}]^{+}$; found 265.9769 .

Potassium (meta-methoxyphenyl)trifluoroborate $\left(\mathrm{K}\left[\mathbf{m}-\mathrm{OMeC}_{6} \mathbf{H}_{4} \mathrm{BF}_{3}\right]\right)$ : yield: $94 \%$, white solid. ${ }^{1} \mathrm{H}$ NMR (400 MHz, $\left.\mathrm{CD}_{3} \mathrm{CN}\right): \sigma 3.72\left(\mathrm{~s}, 3 \mathrm{H}, \mathrm{OCH}_{3}\right), 6.66\left(\mathrm{~d}, 1 \mathrm{H},{ }^{3} \mathrm{~J}_{\mathrm{HH}}=7.6 \mathrm{~Hz}\right.$, arom. $\left.\mathrm{H}\right), 7.00-7.02$ $\left(\mathrm{m}, 2 \mathrm{H}\right.$, arom. H), 7.08 (t, $1 \mathrm{H}, J=7.8 \mathrm{~Hz}$, arom. H). ${ }^{13} \mathrm{C} \mathrm{NMR}\left(100 \mathrm{MHz}, \mathrm{CD}_{3} \mathrm{CN}\right): \sigma 55.3\left(\mathrm{~s}, \mathrm{OCH}_{3}\right)$, 112.3 (s), 117.3 (s), 124.8 (s), 128.7 (s), 159.7 (s). ${ }^{19} \mathrm{~F}$ NMR (376 MHz, $\mathrm{CD}_{3} \mathrm{CN}$ ): $\sigma-142.8$ (d, 3F, $\left.{ }^{1} J_{\mathrm{BF}}=56.2 \mathrm{~Hz}, \mathrm{~B} F_{3}\right) .{ }^{11} \mathrm{~B} \mathrm{NMR}\left(128 \mathrm{MHz}, \mathrm{CD}_{3} \mathrm{CN}\right): \sigma 3.86\left(\mathrm{~d},{ }^{1} J_{\mathrm{BF}}=46.9 \mathrm{~Hz}\right)$. HRMS $(\mathrm{FAB}), m / z$ : calcd. for $\mathrm{C}_{7} \mathrm{H}_{7} \mathrm{OBF}_{3} \mathrm{~K}_{2}{ }^{+} 252.9811[\mathrm{M}+\mathrm{K}]^{+}$; found 252.9818. Elemental analysis calcd. for $\mathrm{C}_{7} \mathrm{H}_{7} \mathrm{OBF}_{3} \mathrm{~K}: \mathrm{C}, 39.28 ; \mathrm{H}, 3.30$; found: $\mathrm{C}, 38.71 ; \mathrm{H}, 3.27$.

Potassium (meta-ethoxyphenyl)trifluoroborate $\left(\mathbf{K}\left[\boldsymbol{m}-\mathbf{O E t C} \mathbf{C}_{6} \mathbf{H}_{\mathbf{4}} \mathbf{B F}_{3}\right]\right)$ : yield: $87 \%$, white solid. ${ }^{1} \mathrm{H}$ NMR (400 MHz, CD $\left.\mathrm{CD}_{3} \mathrm{CN}\right): \sigma 1.30\left(\mathrm{t}, 3 \mathrm{H},{ }^{3} J_{\mathrm{HH}}=7.1 \mathrm{~Hz}, \mathrm{OCH}_{2} \mathrm{CH}_{3}\right), 3.97\left(\mathrm{q}, 2 \mathrm{H},{ }^{3} J_{\mathrm{HH}}=7.1 \mathrm{~Hz}\right.$, $\left.\mathrm{OCH}_{2} \mathrm{CH}_{3}\right), 6.64\left(\mathrm{~d}, 1 \mathrm{H},{ }^{3} J_{\mathrm{HH}}=8.4 \mathrm{~Hz}\right.$, arom. H), 6.98-7.01 (m, 2H, arom. H), $7.07(\mathrm{t}, 1 \mathrm{H}, J=7.8$ $\mathrm{Hz}$, arom. H). ${ }^{13} \mathrm{C} \mathrm{NMR}\left(100 \mathrm{MHz}, \mathrm{CD}_{3} \mathrm{CN}\right): \sigma 15.3\left(\mathrm{~s}, \mathrm{OCH}_{2} \mathrm{CH}_{3}\right), 63.6\left(\mathrm{~s}, \mathrm{OCH}_{2} \mathrm{CH}_{3}\right), 113.0$ (s), 117.9 (s), 124.7 (s), 128.7 (s), 159.0 (s). ${ }^{19} \mathrm{~F}$ NMR (376 MHz, $\mathrm{CD}_{3} \mathrm{CN}$ ): $\sigma-142.7$ (br, 3F, $\left.\mathrm{B} F_{3}\right) .{ }^{11} \mathrm{~B}$ NMR (128 MHz, $\mathrm{CD}_{3} \mathrm{CN}$ ): $\sigma 4.02$ (br). HRMS (FAB), $m / z$ : calcd. for $\mathrm{C}_{8} \mathrm{H}_{9} \mathrm{OBF}_{3} \mathrm{~K}_{2}{ }^{+} 266.9967[\mathrm{M}+$ $\mathrm{K}]^{+}$; found 266.9975. Elemental analysis calcd. for $\mathrm{C}_{8} \mathrm{H}_{9} \mathrm{OBF}_{3} \mathrm{~K}: \mathrm{C}, 42.13$; H, 3.98; found: C, 42.00; H, 3.98 .

Potassium (meta-n-propoxyphenyl)trifluoroborate $\left(\mathbf{K}\left[m-\mathbf{O}^{n} \operatorname{PrC}_{6} \mathbf{H}_{4} \mathbf{B F}_{3}\right]\right)$ : yield: $90 \%$, white 
solid. ${ }^{1} \mathrm{H}$ NMR (400 MHz, $\left.\mathrm{CD}_{3} \mathrm{CN}\right): \sigma 0.98\left(\mathrm{t}, 3 \mathrm{H},{ }^{3} \mathrm{~J}_{\mathrm{HH}}=7.3 \mathrm{~Hz}, \mathrm{OCH}_{2} \mathrm{CH}_{2} \mathrm{CH}_{3}\right), 1.71(\mathrm{~m}, 2 \mathrm{H}, J=$ $\left.7.1 \mathrm{~Hz}, \mathrm{OCH}_{2} \mathrm{CH}_{2} \mathrm{CH}_{3}\right), 3.88\left(\mathrm{t}, 2 \mathrm{H},{ }^{3} J_{\mathrm{HH}}=6.4 \mathrm{~Hz}, \mathrm{OCH}_{2} \mathrm{CH}_{2} \mathrm{CH}_{3}\right), 6.66\left(\mathrm{~d}, 1 \mathrm{H},{ }^{3} J_{\mathrm{HH}}=6.9 \mathrm{~Hz}\right.$, arom. $\mathrm{H}), 7.00-7.02$ (m, 2H, arom. H), 7.08 (t, $1 \mathrm{H}, J=7.6 \mathrm{~Hz}$, arom. H). ${ }^{13} \mathrm{C}$ NMR $\left(100 \mathrm{MHz}, \mathrm{CD}_{3} \mathrm{CN}\right): \sigma$ $10.9\left(\mathrm{~s}, \mathrm{OCH}_{2} \mathrm{CH}_{2} \mathrm{CH}_{3}\right), 23.5\left(\mathrm{~s}, \mathrm{OCH}_{2} \mathrm{CH}_{2} \mathrm{CH}_{3}\right), 69.8$ (s, $\left.\mathrm{OCH}_{2} \mathrm{CH}_{2} \mathrm{CH}_{3}\right), 113.0$ (s), 118.0 (s), 124.7 (s), 128.8 (s), 159.2 (s). ${ }^{19} \mathrm{~F}$ NMR (376 MHz, $\mathrm{CD}_{3} \mathrm{CN}$ ): $\sigma-142.7$ (br, 3F, B $\left.F_{3}\right) .{ }^{11} \mathrm{~B}$ NMR (128 MHz, $\mathrm{CD}_{3} \mathrm{CN}$ ): $\sigma 4.09$ (br). HRMS (FAB), $m / z$ : calcd. for $\mathrm{C}_{9} \mathrm{H}_{11} \mathrm{OBF}_{3} \mathrm{~K}_{2}{ }^{+} 281.0124[\mathrm{M}+\mathrm{K}]^{+}$; found 281.0133. Elemental analysis calcd. for $\mathrm{C}_{9} \mathrm{H}_{11} \mathrm{OBF}_{3} \mathrm{~K}$ : C, 44.65; H, 4.58; found: $\mathrm{C}, 44.43$; H, 4.57 .

Potassium (meta-iso-propoxyphenyl)trifluoroborate $\left(\mathrm{K}\left[m-\mathrm{O}^{i} \mathrm{PrC}_{6} \mathbf{H}_{\mathbf{4}} \mathrm{BF}_{3}\right]\right)$ : yield: $74 \%$, white solid. ${ }^{1} \mathrm{H}$ NMR (400 MHz, $\left.\mathrm{CD}_{3} \mathrm{CN}\right): \sigma 1.23\left(\mathrm{~d}, 6 \mathrm{H},{ }^{3} J_{\mathrm{HH}}=6.0 \mathrm{~Hz}, \mathrm{OCH}\left(\mathrm{CH}_{3}\right)_{2}\right), 4.54(\mathrm{~m}, 1 \mathrm{H}, J=6.1$ $\left.\mathrm{Hz}, \mathrm{OCH}\left(\mathrm{CH}_{3}\right)_{2}\right), 6.65\left(\mathrm{td}, 1 \mathrm{H},{ }^{3} J_{\mathrm{HH}}=7.8 \mathrm{~Hz},{ }^{4} \mathrm{~J}=1.4 \mathrm{~Hz}\right.$, arom. H), 6.97-7.01 (m, 2H, arom. H), $7.07\left(\mathrm{t}, 1 \mathrm{H}, J=7.6 \mathrm{~Hz}\right.$, arom. H). ${ }^{13} \mathrm{C} \mathrm{NMR}\left(100 \mathrm{MHz}, \mathrm{CD}_{3} \mathrm{CN}\right): \sigma 22.5\left(\mathrm{~s}, \mathrm{OCH}\left(\mathrm{CH}_{3}\right)_{2}\right), 69.9(\mathrm{~s}$, $\left.\mathrm{OCH}\left(\mathrm{CH}_{3}\right)_{2}\right), 114.5$ (s), 119.6 (s), 124.7 (s), 128.8 (s), 157.9 (s). ${ }^{19} \mathrm{~F}$ NMR (376 MHz, $\left.\mathrm{CD}_{3} \mathrm{CN}\right): \sigma$ -142.7 (br, 3F, BF $).{ }^{11} \mathrm{~B}$ NMR (128 MHz, $\mathrm{CD}_{3} \mathrm{CN}$ ): $\sigma 4.08$ (br). HRMS (FAB), $m / z$ : calcd. for $\mathrm{C}_{9} \mathrm{H}_{11} \mathrm{OBF}_{3} \mathrm{~K}_{2}{ }^{+} 281.0124[\mathrm{M}+\mathrm{K}]^{+}$; found 281.0131. Elemental analysis calcd. for $\mathrm{C}_{9} \mathrm{H}_{11} \mathrm{OBF}_{3} \mathrm{~K}: \mathrm{C}$, 44.65; H, 4.58; found: C, 43.87; H, 4.71.

Potassium (meta-n-butoxyphenyl)trifluoroborate $\left(\mathrm{K}\left[m-\mathbf{O}^{n} \mathbf{B t C}_{6} \mathbf{H}_{4} \mathbf{B F}_{3}\right]\right)$ : yield: $73 \%$, white solid. ${ }^{1} \mathrm{H}$ NMR (400 MHz, $\mathrm{CD}_{3} \mathrm{CN}$ ): $\sigma 0.93\left(\mathrm{t}, 3 \mathrm{H},{ }^{3} \mathrm{~J}_{\mathrm{HH}}=7.3 \mathrm{~Hz}, \mathrm{OCH}_{2} \mathrm{CH}_{2} \mathrm{CH}_{2} \mathrm{CH}_{3}\right), 1.39-1.49(\mathrm{~m}, 2 \mathrm{H}$, $\left.\mathrm{OCH}_{2} \mathrm{CH}_{2} \mathrm{CH}_{2} \mathrm{CH}_{3}\right), \quad 1.64-1.71\left(\mathrm{~m}, 2 \mathrm{H}, \quad \mathrm{OCH}_{2} \mathrm{CH}_{2} \mathrm{CH}_{2} \mathrm{CH}_{3}\right), 3.92\left(\mathrm{t}, 2 \mathrm{H},{ }^{3} J_{\mathrm{HH}}=6.6 \mathrm{~Hz}\right.$, $\left.\mathrm{OCH}_{2} \mathrm{CH}_{2} \mathrm{CH}_{2} \mathrm{CH}_{3}\right), 6.63\left(\mathrm{dd}, 1 \mathrm{H},{ }^{3} J_{\mathrm{HH}}=7.8 \mathrm{~Hz},{ }^{4} \mathrm{~J}=0.92 \mathrm{~Hz}\right.$, arom. H), 6.97-6.99 (m, 2H, arom. $\mathrm{H}), 7.06$ (t, $1 \mathrm{H}, J=7.3 \mathrm{~Hz}$, arom. H). ${ }^{13} \mathrm{C} \mathrm{NMR}\left(100 \mathrm{MHz}, \mathrm{CD}_{3} \mathrm{CN}\right): \sigma 14.2\left(\mathrm{~s}, \mathrm{OCH}_{2} \mathrm{CH}_{2} \mathrm{CH}_{2} \mathrm{CH}_{3}\right)$, $20.0\left(\mathrm{~s}, \mathrm{OCH}_{2} \mathrm{CH}_{2} \mathrm{CH}_{2} \mathrm{CH}_{3}\right), 32.3\left(\mathrm{~s}, \mathrm{OCH}_{2} \mathrm{CH}_{2} \mathrm{CH}_{2} \mathrm{CH}_{3}\right), 67.9$ (s, $\mathrm{OCH}_{2} \mathrm{CH}_{2} \mathrm{CH}_{2} \mathrm{CH}_{3}$ ), 112.9 (s), 117.9 (s), 124.7 (s), 128.7 (s), 159.2 (s). ${ }^{19} \mathrm{~F}$ NMR (376 MHz, $\mathrm{CD}_{3} \mathrm{CN}$ ): $\sigma-142.7$ (br, 3F, $\left.\mathrm{B} F_{3}\right) .{ }^{11} \mathrm{~B}$ NMR (128 MHz, $\mathrm{CD}_{3} \mathrm{CN}$ ): $\sigma 3.80$ (br). HRMS (FAB), $m / z$ : calcd. for $\mathrm{C}_{10} \mathrm{H}_{13} \mathrm{OBF}_{3} \mathrm{~K}_{2}{ }^{+} 295.0280$ [M $+\mathrm{K}]^{+}$; found 295.0296. Elemental analysis calcd. for $\mathrm{C}_{10} \mathrm{H}_{13} \mathrm{OBF}_{3} \mathrm{~K}: \mathrm{C}, 46.90$; $\mathrm{H}, 5.12$; found: $\mathrm{C}$, 46.87; H, 5.19.

Potassium (meta-n-decyloxyphenyl)trifluoroborate $\left(\mathrm{K}\left[m-\mathrm{O}\left(\mathrm{CH}_{2}\right)_{9} \mathrm{CH}_{3} \mathrm{C}_{6} \mathrm{H}_{4} \mathrm{BF}_{3}\right]\right)$ : yield: $46 \%$, white solid. ${ }^{1} \mathrm{H}$ NMR (400 MHz, $\left.\mathrm{CD}_{3} \mathrm{CN}\right): \sigma 0.85\left(\mathrm{t}, 3 \mathrm{H},{ }^{3} J_{\mathrm{HH}}=6.9 \mathrm{~Hz}, \mathrm{O}\left(\mathrm{CH}_{2}\right){ }_{9} \mathrm{CH}_{3}\right), 1.26-1.44(\mathrm{~m}$, $\left.14 \mathrm{H}, \mathrm{OCH}_{2} \mathrm{CH}_{2}\left(\mathrm{CH}_{2}\right)_{7} \mathrm{CH}_{3}\right), 1.65-1.72\left(\mathrm{~m}, 2 \mathrm{H}, \mathrm{OCH}_{2} \mathrm{CH}_{2}\left(\mathrm{CH}_{2}\right)_{7} \mathrm{CH}_{3}\right), 3.90\left(\mathrm{t}, 2 \mathrm{H},{ }^{3} J_{\mathrm{HH}}=6.4 \mathrm{~Hz}\right.$, $\left.\mathrm{OCH}_{2}\left(\mathrm{CH}_{2}\right)_{8} \mathrm{CH}_{3}\right), 6.62\left(\mathrm{dd}, 1 \mathrm{H},{ }^{3} J_{\mathrm{HH}}=8.0 \mathrm{~Hz},{ }^{4} \mathrm{~J}=2.4 \mathrm{~Hz}\right.$, arom. H), 6.95-6.98 (m, 2H, arom. H), 7.05 (t, $1 \mathrm{H}, J=7.6 \mathrm{~Hz}$, arom. H). ${ }^{13} \mathrm{C} \mathrm{NMR}\left(100 \mathrm{MHz}, \mathrm{CD}_{3} \mathrm{CN}\right): \sigma 14.4\left(\mathrm{~s}, \mathrm{O}\left(\mathrm{CH}_{2}\right)_{9} \mathrm{CH}_{3}\right), 23.4(\mathrm{~s}$, $\left.\mathrm{O}\left(\mathrm{CH}_{2}\right)_{8} \mathrm{CH}_{2} \mathrm{CH}_{3}\right), 26.8$ (s, O $\left.\left(\mathrm{CH}_{2}\right)_{7} \mathrm{CH}_{2} \mathrm{CH}_{2} \mathrm{CH}_{3}\right), 30.0-30.4$ (m, 5C, $\mathrm{OCH}_{2} \mathrm{CH}_{2}\left(\mathrm{CH}_{2}\right)_{5} \mathrm{CH}_{2} \mathrm{CH}_{2} \mathrm{CH}_{3}$ ), $32.6\left(\mathrm{~s}, \mathrm{OCH}_{2} \mathrm{CH}_{2}\left(\mathrm{CH}_{2}\right)_{7} \mathrm{CH}_{3}\right), 68.2\left(\mathrm{~s}, \mathrm{OCH}_{2}\left(\mathrm{CH}_{2}\right)_{8} \mathrm{CH}_{3}\right), 112.8$ (s), 117.9 (s), 124.7 (s), 128.6 (s), 159.2 (s). ${ }^{19} \mathrm{~F}$ NMR (376 MHz, $\mathrm{CD}_{3} \mathrm{CN}$ ): $\sigma-142.7$ (br, 3F, B $\left.F_{3}\right) .{ }^{11} \mathrm{~B}$ NMR $\left(128 \mathrm{MHz}, \mathrm{CD}_{3} \mathrm{CN}\right): \sigma$ 3.88 (br). HRMS (FAB), $m / z$ : calcd. for $\mathrm{C}_{16} \mathrm{H}_{25} \mathrm{OBF}_{3} \mathrm{~K}_{2}{ }^{+} 379.1219[\mathrm{M}+\mathrm{K}]^{+}$; found 379.1217 . Elemental analysis calcd. for $\mathrm{C}_{16} \mathrm{H}_{25} \mathrm{OBF}_{3} \mathrm{~K}$ : C, 56.48; $\mathrm{H}, 7.41$; found: $\mathrm{C}, 56.18$; H, 7.61. 
Potassium (meta-(2-methoxyethoxy)phenyl)trifluoroborate $\left(\mathrm{K}\left[m-\mathrm{O}\left(\mathrm{CH}_{2}\right)_{2} \mathrm{OCH}_{3} \mathrm{C}_{6} \mathrm{H}_{4} \mathrm{BF}_{3}\right]\right)$ : yield: $47 \%$, white solid. ${ }^{1} \mathrm{H}$ NMR (400 MHz, $\left.\mathrm{CD}_{3} \mathrm{CN}\right): \sigma 3.32\left(\mathrm{~s}, 3 \mathrm{H}, \mathrm{O}\left(\mathrm{CH}_{2}\right)_{2} \mathrm{OCH}_{3}\right), 3.63(\mathrm{t}, 2 \mathrm{H}$, $\left.{ }^{3} J_{\mathrm{HH}}=64.8 \mathrm{~Hz}, \mathrm{OCH}_{2} \mathrm{CH}_{2} \mathrm{OCH}_{3}\right), 4.03\left(\mathrm{t}, 2 \mathrm{H},{ }^{3} J_{\mathrm{HH}}=4.8 \mathrm{~Hz}, \mathrm{OCH}_{2} \mathrm{CH}_{2} \mathrm{OCH}_{3}\right), 6.64\left(\mathrm{dd}, 1 \mathrm{H},{ }^{3} J_{\mathrm{HH}}=\right.$ $7.8 \mathrm{~Hz},{ }^{4} J=2.3 \mathrm{~Hz}$, arom. H), 6.97-7.00 (m, 2H, arom. H), $7.06\left(\mathrm{t}, 1 \mathrm{H}, J=7.8 \mathrm{~Hz}\right.$, arom. H). ${ }^{13} \mathrm{C}$ NMR (100 MHz, CD ${ }_{3} \mathrm{CN}$ ): $\sigma 59.0$ (s), 67.6 (s), 72.0 (s), 112.9 (s), 117.7 (s), 125.0 (s), 128.7 (s), $158.8(\mathrm{~s}) .{ }^{19} \mathrm{~F}$ NMR $\left(376 \mathrm{MHz}, \mathrm{CD}_{3} \mathrm{CN}\right): \sigma-142.8\left(\mathrm{~d}, 3 \mathrm{~F},{ }^{1} J_{\mathrm{BF}}=53.2 \mathrm{~Hz}, \mathrm{~B} F_{3}\right) .{ }^{11} \mathrm{~B} \mathrm{NMR}(128 \mathrm{MHz}$, $\left.\mathrm{CD}_{3} \mathrm{CN}\right): \sigma 3.71\left(\mathrm{~d},{ }^{1} J_{\mathrm{BF}}=46.9 \mathrm{~Hz}\right)$. HRMS (FAB), $m / z$ : calcd. for $\mathrm{C}_{9} \mathrm{H}_{11} \mathrm{O}_{2} \mathrm{BF}_{3} \mathrm{~K}_{2}{ }^{+} 297.0073[\mathrm{M}+$ $\mathrm{K}]^{+}$; found 297.0084 .

Cesium phenyltrifluoroborate $\left(\mathbf{C s}\left[\mathbf{P h B F}_{3}\right]\right)$ : yield: $89 \%$, white solid. ${ }^{1} \mathrm{H}$ NMR (400 $\mathrm{MHz}$, $\left.\mathrm{CD}_{3} \mathrm{CN}\right): \sigma 7.09\left(\mathrm{t}, 1 \mathrm{H}, J=8.0 \mathrm{~Hz}\right.$, arom. H), $7.15\left(\mathrm{t}, 2 \mathrm{H}, J=7.3 \mathrm{~Hz}\right.$, arom. H), $7.41\left(\mathrm{~d}, 2 \mathrm{H},{ }^{3} J_{\mathrm{HH}}=\right.$ $6.9 \mathrm{~Hz}$, arom. H). $\left.{ }^{13} \mathrm{C} \mathrm{NMR} \mathrm{(100} \mathrm{MHz,} \mathrm{CD}_{3} \mathrm{CN}\right): \sigma 126.5$ (s), 127.7 (s), 132.3 (s). ${ }^{19} \mathrm{~F}$ NMR (376 $\left.\mathrm{MHz}, \mathrm{CD}_{3} \mathrm{CN}\right): \sigma-139.0\left(\mathrm{q}, 3 \mathrm{~F},{ }^{1} J_{\mathrm{BF}}=51.4 \mathrm{~Hz}, \mathrm{~B} F_{3}\right) .{ }^{11} \mathrm{~B} \mathrm{NMR}\left(128 \mathrm{MHz}, \mathrm{CD}_{3} \mathrm{CN}\right): \sigma 3.81\left(\mathrm{q},{ }^{1} J_{\mathrm{BF}}\right.$ $=56.2 \mathrm{~Hz}$ ). HRMS (FAB), $m / z$ : calcd. for $\mathrm{C}_{6} \mathrm{H}_{5} \mathrm{BF}_{3} \mathrm{Cs}_{2}{ }^{+} 410.8540[\mathrm{M}+\mathrm{Cs}]^{+}$; found 410.8548 . Elemental analysis calcd. for $\mathrm{C}_{6} \mathrm{H}_{5} \mathrm{BF}_{3} \mathrm{Cs}$ : C, 25.94; H, 1.81; found: C, 25.27; H, 2.04.

Cesium (meta-trifluoromethylphenyl)trifluoroborate $\left(\mathbf{C s}\left[m-\mathbf{C F}_{3} \mathbf{C}_{6} \mathbf{H}_{\mathbf{4}} \mathbf{B} \mathbf{F}_{3}\right]\right)$ : yield: $99 \%$, white solid. ${ }^{1} \mathrm{H}$ NMR (400 MHz, $\left.\mathrm{CD}_{3} \mathrm{CN}\right): \sigma 7.33\left(\mathrm{t}, 1 \mathrm{H}, J=7.5 \mathrm{~Hz}\right.$, arom. H), $7.41\left(\mathrm{~d}, 1 \mathrm{H},{ }^{3} J_{\mathrm{HH}}=7.8 \mathrm{~Hz}\right.$ ), 7.66-7.70 (m, 2H, arom. H). $\left.{ }^{13} \mathrm{C} \mathrm{NMR} \mathrm{(100} \mathrm{MHz,} \mathrm{CD}_{3} \mathrm{CN}\right): \sigma 123.1(\mathrm{~s}), 126.4\left(\mathrm{q},{ }^{1} J_{\mathrm{CF}}=269.8 \mathrm{~Hz}\right.$, $\left.C_{3}\right), 128.1(\mathrm{~s}), 128.4(\mathrm{~s}), 129.0\left(\mathrm{q},{ }^{2} J_{\mathrm{CF}}=29.6 \mathrm{~Hz}, C \mathrm{CF}_{3}\right), 136.1(\mathrm{~s}) .{ }^{19} \mathrm{~F} \mathrm{NMR}\left(376 \mathrm{MHz}, \mathrm{CD}_{3} \mathrm{CN}\right)$ : $\sigma-140.1$ (br, 3F, B $F_{3}$ ), -63.1 (s, 3F, $\mathrm{CF}_{3}$ ). ${ }^{11} \mathrm{~B} \mathrm{NMR}\left(128 \mathrm{MHz}, \mathrm{CD}_{3} \mathrm{CN}\right): \sigma 3.46\left(\mathrm{q},{ }^{1} J_{\mathrm{BF}}=51.8 \mathrm{~Hz}\right.$ ). HRMS (FAB), $m / z$ : calcd. for $\mathrm{C}_{7} \mathrm{H}_{4} \mathrm{BF}_{6} \mathrm{Cs}_{2}{ }^{+} 478.8414[\mathrm{M}+\mathrm{Cs}]^{+}$; found 478.8412. Elemental analysis calcd. for $\mathrm{C}_{6} \mathrm{H}_{4} \mathrm{BF}_{4} \mathrm{Cs}$ : C, 24.31; $\mathrm{H}, 1.17$; found: $\mathrm{C}, 24.10 ; \mathrm{H}, 1.18$.

Cesium (meta-fluorophenyl)trifluoroborate $\left(\mathbf{C s}\left[\boldsymbol{m}-\mathbf{F C}_{\mathbf{6}} \mathbf{H}_{\mathbf{4}} \mathbf{B} \mathbf{F}_{3}\right]\right)$ : yield: $99 \%$, white solid. ${ }^{1} \mathrm{H}$ NMR (400 MHz, $\left.\mathrm{CD}_{3} \mathrm{CN}\right): \sigma 6.78-6.83\left(\mathrm{~m}, 1 \mathrm{H}\right.$, arom. H), 7.08-7.22 (m, 3H, arom. H). ${ }^{13} \mathrm{C}$ NMR $\left(100 \mathrm{MHz}, \mathrm{CD}_{3} \mathrm{CN}\right): \sigma 112.8\left(\mathrm{~d},{ }^{2} J_{\mathrm{CF}}=21.0 \mathrm{~Hz}\right), 118.0\left(\mathrm{~d},{ }^{2} J_{\mathrm{CF}}=16.2 \mathrm{~Hz}\right), 128.0(\mathrm{~s}), 129.4\left(\mathrm{~d},{ }^{3} J_{\mathrm{CF}}=\right.$ $5.7 \mathrm{~Hz}), 163.5\left(\mathrm{~d},{ }^{1} J_{\mathrm{CF}}=240.3 \mathrm{~Hz}, C \mathrm{~F}\right) .{ }^{19} \mathrm{~F} \mathrm{NMR}\left(376 \mathrm{MHz}, \mathrm{CD}_{3} \mathrm{CN}\right): \sigma-139.8\left(\mathrm{q}, 3 \mathrm{~F},{ }^{1} J_{\mathrm{BF}}=48.4\right.$ $\left.\mathrm{Hz}, \mathrm{B} F_{3}\right),-118.1(\mathrm{~s}, 1 \mathrm{~F}, 3-F) .{ }^{11} \mathrm{~B} \mathrm{NMR}\left(128 \mathrm{MHz}, \mathrm{CD}_{3} \mathrm{CN}\right): \sigma 3.37$ (q, $\left.{ }^{1} J_{\mathrm{BF}}=52.8 \mathrm{~Hz}\right)$. HRMS (FAB), $m / z$ : calcd. for $\mathrm{C}_{6} \mathrm{H}_{4} \mathrm{BF}_{4} \mathrm{Cs}_{2}{ }^{+} 428.8446[\mathrm{M}+\mathrm{Cs}]^{+}$; found 428.8453. Elemental analysis calcd. for $\mathrm{C}_{6} \mathrm{H}_{4} \mathrm{BF}_{4} \mathrm{Cs}$ : C, 24.36; H, 1.36; found: C, 23.73; H, 1.60 .

Cesium (meta-trifluoromethoxyphenyl)trifluoroborate $\left(\mathrm{Cs}\left[m-\mathrm{OCF}_{3} \mathbf{C}_{6} \mathbf{H}_{\mathbf{4}} \mathrm{BF}_{3}\right]\right)$ : yield: $62 \%$, white solid. ${ }^{1} \mathrm{H}$ NMR (400 MHz, $\left.\mathrm{CD}_{3} \mathrm{CN}\right): \sigma 7.00\left(\mathrm{~d}, 1 \mathrm{H},{ }^{3} J_{\mathrm{HH}}=8.0 \mathrm{~Hz}\right.$, arom. H), $7.24(\mathrm{t}, 1 \mathrm{H}, J=$ $7.8 \mathrm{~Hz}$, arom. H), $7.28\left(\mathrm{~s}, 1 \mathrm{H}\right.$, arom. H), $7.40\left(\mathrm{~d}, 1 \mathrm{H},{ }^{3} J_{\mathrm{HH}}=7.3 \mathrm{~Hz}\right.$, arom. H). ${ }^{13} \mathrm{C} \mathrm{NMR} \mathrm{(100} \mathrm{MHz,}$ $\mathrm{CD}_{3} \mathrm{CN}$ ): $\sigma 119.0(\mathrm{~s}), 121.7\left(\mathrm{q},{ }^{1} J_{\mathrm{CF}}=252.4 \mathrm{~Hz}, \mathrm{OCF}_{3}\right), 124.1(\mathrm{~s}), 129.3(\mathrm{~s}), 131.1(\mathrm{~s}), 149.5(\mathrm{~s}) .{ }^{19} \mathrm{~F}$ NMR $\left(376 \mathrm{MHz}, \mathrm{CD}_{3} \mathrm{CN}\right): \sigma-139.8\left(\mathrm{q}, 3 \mathrm{~F},{ }^{1} J_{\mathrm{BF}}=57.8 \mathrm{~Hz}, \mathrm{~B} F_{3}\right),-58.6\left(\mathrm{~s}, 3 \mathrm{~F}, \mathrm{OC} F_{3}\right) .{ }^{11} \mathrm{~B} \mathrm{NMR}$ 
$\left(128 \mathrm{MHz}, \mathrm{CD}_{3} \mathrm{CN}\right): \sigma 3.35\left(\mathrm{q},{ }^{1} J_{\mathrm{BF}}=50.8 \mathrm{~Hz}\right)$. HRMS (FAB), $m / z$ : calcd. for $\mathrm{C}_{7} \mathrm{H}_{4} \mathrm{OBF}_{6} \mathrm{Cs}_{2}{ }^{+}$ 494.8363 [M $+\mathrm{Cs}]^{+}$; found 494.8367. Elemental analysis calcd. for $\mathrm{C}_{7} \mathrm{H}_{4} \mathrm{OBF}_{6} \mathrm{Cs}: \mathrm{C}, 23.24 ; \mathrm{H}, 1.11$; found: C, 23.07; H, 1.14.

Cesium (meta-methylthiophenyl)trifluoroborate $\left(\mathrm{Cs}\left[m-\mathrm{SMeC}_{6} \mathbf{H}_{4} \mathbf{B F}_{3}\right]\right)$ : yield: $94 \%$, pale brown solid. ${ }^{1} \mathrm{H}$ NMR (400 MHz, $\left.\mathrm{CD}_{3} \mathrm{CN}\right): \sigma 2.41(\mathrm{~s}, 3 \mathrm{H}, \mathrm{SCH}), 7.02\left(\mathrm{dd}, 1 \mathrm{H},{ }^{3} J_{\mathrm{HH}}=7.8 \mathrm{~Hz},{ }^{4} \mathrm{~J}=0.92 \mathrm{~Hz}\right.$, arom. H), 7.10 (t, $1 \mathrm{H}, J=7.3 \mathrm{~Hz}$, arom. H), $7.20\left(\mathrm{~d}, 1 \mathrm{H},{ }^{3} J_{\mathrm{HH}}=6.9 \mathrm{~Hz}\right.$, arom. H), 7.33 (s, 1H, arom. H). ${ }^{13} \mathrm{C}$ NMR (100 MHz, $\left.\mathrm{CD}_{3} \mathrm{CN}\right): \sigma 16.0\left(\mathrm{~s}, \mathrm{SCH}_{3}\right), 125.0$ (s), 128.4 (s), 129.3 (s), 130.6 (s), 136.8 (s). ${ }^{19} \mathrm{~F}$ NMR (376 MHz, $\left.\mathrm{CD}_{3} \mathrm{CN}\right): \sigma-139.2\left(\mathrm{br}, 3 \mathrm{~F}, \mathrm{~B} F_{3}\right) .{ }^{11} \mathrm{~B} \mathrm{NMR}\left(128 \mathrm{MHz}, \mathrm{CD}_{3} \mathrm{CN}\right): \sigma 3.60$ (q, ${ }^{1} J_{\mathrm{BF}}=52.5 \mathrm{~Hz}$ ). HRMS (FAB), $m / z$ : calcd. for $\mathrm{C}_{7} \mathrm{H}_{7} \mathrm{SBF}_{3} \mathrm{Cs}_{2}{ }^{+} 456.8417[\mathrm{M}+\mathrm{Cs}]^{+}$; found 456.8427 . Elemental analysis calcd. for $\mathrm{C}_{7} \mathrm{H}_{7} \mathrm{SBF}_{3} \mathrm{Cs}$ : C, 25.96; H, 2.18; found: C, 26.18; H, 2.27.

Cesium (meta-ethoxyphenyl)trifluoroborate $\left(\mathbf{C s}\left[\boldsymbol{m}-\mathbf{O E t C}_{\mathbf{6}} \mathbf{H}_{\mathbf{4}} \mathbf{B F}_{3}\right]\right)$ : yield: $96 \%$, white solid. ${ }^{1} \mathrm{H}$ NMR (400 MHz, CD $3 \mathrm{CN}): \sigma 1.30\left(\mathrm{t}, 3 \mathrm{H},{ }^{3} J_{\mathrm{HH}}=7.1 \mathrm{~Hz}, \mathrm{OCH}_{2} \mathrm{CH}_{3}\right), 3.98\left(\mathrm{q}, 2 \mathrm{H},{ }^{3} J_{\mathrm{HH}}=6.9 \mathrm{~Hz}\right.$, $\left.\mathrm{OCH}_{2} \mathrm{CH}_{3}\right), 6.62\left(\mathrm{~d}, 1 \mathrm{H},{ }^{3} J_{\mathrm{HH}}=7.8 \mathrm{~Hz}\right.$, arom. H), 6.95-6.98 (m, 2H, arom. H), $7.06(\mathrm{t}, 1 \mathrm{H}, J=7.6$ $\mathrm{Hz}$, arom. H). ${ }^{13} \mathrm{C} \mathrm{NMR}\left(100 \mathrm{MHz}, \mathrm{CD}_{3} \mathrm{CN}\right): \sigma 15.3\left(\mathrm{~s}, \mathrm{OCH}_{2} \mathrm{CH}_{3}\right), 63.6\left(\mathrm{~s}, \mathrm{OCH}_{2} \mathrm{CH}_{3}\right), 112.9$ (s), 117.7 (s), 124.7 (s), 128.7 (s), 159.0 (s). ${ }^{19} \mathrm{~F} \mathrm{NMR} \mathrm{(376} \mathrm{MHz,} \mathrm{CD}_{3} \mathrm{CN}$ ): $\sigma-138.7$ (br, 3F, BF 3 ). ${ }^{11} \mathrm{~B}$ NMR (128 MHz, $\left.\mathrm{CD}_{3} \mathrm{CN}\right): \sigma 3.70\left(\mathrm{~d},{ }^{1} J_{\mathrm{BF}}=48.9 \mathrm{~Hz}\right)$. HRMS (FAB), $m / z$ : calcd. for $\mathrm{C}_{8} \mathrm{H}_{9} \mathrm{OBF}_{3} \mathrm{Cs}_{2}{ }^{+}$ $454.8802[\mathrm{M}+\mathrm{Cs}]^{+}$; found 454.8814. Elemental analysis calcd. for $\mathrm{C}_{8} \mathrm{H}_{9} \mathrm{OBF}_{3} \mathrm{Cs}: \mathrm{C}, 29.85 ; \mathrm{H}, 2.82$; found: C, 29.55; H, 2.97.

Cesium (meta-n-propoxyphenyl)trifluoroborate (Cs[m-O $\left.\left.{ }^{n} \operatorname{PrC}_{6} \mathbf{H}_{4} \mathbf{B F}_{3}\right]\right)$ : yield: $96 \%$, white solid. ${ }^{1} \mathrm{H} \mathrm{NMR}\left(400 \mathrm{MHz}, \mathrm{CD}_{3} \mathrm{CN}\right): \sigma 0.97\left(\mathrm{t}, 3 \mathrm{H},{ }^{3} \mathrm{~J}_{\mathrm{HH}}=7.3 \mathrm{~Hz}, \mathrm{OCH}_{2} \mathrm{CH}_{2} \mathrm{CH}_{3}\right), 1.71(\mathrm{~m}, 2 \mathrm{H}, J=7.1 \mathrm{~Hz}$, $\left.\mathrm{OCH}_{2} \mathrm{CH}_{2} \mathrm{CH}_{3}\right), 3.88\left(\mathrm{t}, 2 \mathrm{H},{ }^{3} J_{\mathrm{HH}}=6.6 \mathrm{~Hz}, \mathrm{OCH}_{2} \mathrm{CH}_{2} \mathrm{CH}_{3}\right), 6.63\left(\mathrm{dd}, 1 \mathrm{H},{ }^{3} J_{\mathrm{HH}}=8.0 \mathrm{~Hz},{ }^{4} \mathrm{~J}=1.6 \mathrm{~Hz}\right.$, arom. H), 6.95-6.98 (m, 2H, arom. H), $7.06\left(\mathrm{t}, 1 \mathrm{H}, J=7.6 \mathrm{~Hz}\right.$, arom. H). ${ }^{13} \mathrm{C} \mathrm{NMR} \mathrm{(100} \mathrm{MHz,}$ $\left.\mathrm{CD}_{3} \mathrm{CN}\right): \sigma 10.8\left(\mathrm{~s}, \mathrm{OCH}_{2} \mathrm{CH}_{2} \mathrm{CH}_{3}\right), 23.5\left(\mathrm{~s}, \mathrm{OCH}_{2} \mathrm{CH}_{2} \mathrm{CH}_{3}\right), 69.7$ (s, $\mathrm{OCH}_{2} \mathrm{CH}_{2} \mathrm{CH}_{3}$ ), 112.9 (s), 117.8 (s), 124.7 (s), 128.7 (s), 159.2 (s). ${ }^{19} \mathrm{~F}$ NMR (376 MHz, $\mathrm{CD}_{3} \mathrm{CN}$ ): $\sigma-138.5$ (br, $\left.3 \mathrm{~F}, \mathrm{~B} F_{3}\right) .{ }^{11} \mathrm{~B}$ NMR (128 MHz, $\left.\mathrm{CD}_{3} \mathrm{CN}\right): \sigma 3.72\left(\mathrm{~d},{ }^{1} J_{\mathrm{BF}}=48.9 \mathrm{~Hz}\right.$ ). HRMS (FAB), $m / z$ : calcd. for $\mathrm{C}_{9} \mathrm{H}_{11} \mathrm{OBF}_{3} \mathrm{Cs}_{2}{ }^{+}$ $468.8959[\mathrm{M}+\mathrm{Cs}]^{+}$; found 468.8966. Elemental analysis calcd. for $\mathrm{C}_{9} \mathrm{H}_{11} \mathrm{OBF}_{3} \mathrm{Cs}: \mathrm{C}, 32.18$; $\mathrm{H}, 3.30$; found: C, 31.49; H, 3.22 .

Potassium (ortho-fluorophenyl)trifluoroborate $\left(\mathbf{K}\left[\boldsymbol{o}-\mathbf{F C}_{\mathbf{6}} \mathbf{H}_{\mathbf{4}} \mathbf{B} \mathbf{F}_{3}\right]\right)$ : yield: $79 \%$, white solid. ${ }^{1} \mathrm{H}$ NMR (400 MHz, $\mathrm{CD}_{3} \mathrm{CN}$ ): $\sigma 6.83$ (t, $1 \mathrm{H}, J=8.9 \mathrm{~Hz}$, arom. H), 6.97 (t, $1 \mathrm{H}, J=7.3 \mathrm{~Hz}$, arom. H), $7.10-7.15\left(\mathrm{~m}, 1 \mathrm{H}\right.$, arom. H), $7.42\left(\mathrm{t}, 1 \mathrm{H}, J=6.2 \mathrm{~Hz}\right.$, arom. H). ${ }^{13} \mathrm{C} \mathrm{NMR}\left(100 \mathrm{MHz}, \mathrm{CD}_{3} \mathrm{CN}\right): \sigma$ $114.7\left(\mathrm{~d},{ }^{2} J_{\mathrm{CF}}=25.9 \mathrm{~Hz}\right), 123.9(\mathrm{~s}), 128.8\left(\mathrm{~d},{ }^{3} J_{\mathrm{CF}}=7.7 \mathrm{~Hz}\right), 135.0\left(\mathrm{~d},{ }^{3} J_{\mathrm{CF}}=10.5 \mathrm{~Hz}\right), 167.0\left(\mathrm{~d},{ }^{1} J_{\mathrm{BF}}\right.$ $=237.6 \mathrm{~Hz}) \cdot{ }^{19} \mathrm{~F}$ NMR $\left(376 \mathrm{MHz}, \mathrm{CD}_{3} \mathrm{CN}\right): \sigma-140.8\left(\mathrm{q}, 3 \mathrm{~F},{ }^{1} J_{\mathrm{BF}}=49.6 \mathrm{~Hz}, \mathrm{~B} F_{3}\right),-110.8(\mathrm{~s}, 1 \mathrm{~F}$, $2-F) .{ }^{11} \mathrm{~B}$ NMR $\left(128 \mathrm{MHz}, \mathrm{CD}_{3} \mathrm{CN}\right): \sigma 3.18\left(\mathrm{q},{ }^{1} J_{\mathrm{BF}}=51.3 \mathrm{~Hz}\right.$ ). HRMS (FAB), $m / z$ : calcd. for $\mathrm{C}_{6} \mathrm{H}_{4} \mathrm{BF}_{4} \mathrm{~K}_{2}^{+} 240.9611[\mathrm{M}+\mathrm{K}]^{+}$; found 240.9613. Elemental analysis calcd. for $\mathrm{C}_{6} \mathrm{H}_{4} \mathrm{BF}_{4} \mathrm{~K}$ : $\mathrm{C}$, 
35.68; H, 2.00; found: C, 34.92; H, 1.99 .

Potassium (ortho-trifluoromethoxyphenyl)trifluoroborate $\left(\mathrm{K}\left[\boldsymbol{o}-\mathrm{OCF}_{3} \mathbf{C}_{6} \mathrm{H}_{4} \mathrm{BF}_{3}\right]\right.$ ): yield: $80 \%$, white solid. ${ }^{1} \mathrm{H}$ NMR $\left(400 \mathrm{MHz}, \mathrm{CD}_{3} \mathrm{CN}\right): \sigma 7.07$ (d, $1 \mathrm{H},{ }^{3} \mathrm{~J}_{\mathrm{HH}}=7.8 \mathrm{~Hz}$, arom. H), 7.12-7.20 (m, 2H, arom. H), $7.53\left(\mathrm{~d}, 1 \mathrm{H},{ }^{3} J_{\mathrm{HH}}=6.9 \mathrm{~Hz}\right.$, arom. H). ${ }^{13} \mathrm{C} \mathrm{NMR}\left(100 \mathrm{MHz}, \mathrm{CD}_{3} \mathrm{CN}\right): \sigma 119.9$ (s), 121.9 (q, $\left.{ }^{1} J_{\mathrm{CF}}=253.9 \mathrm{~Hz}, \mathrm{OCF}_{3}\right), 126.7$ (s), $128.4(\mathrm{~s}), 135.3$ (s), 153.5 (s). ${ }^{19} \mathrm{~F}$ NMR (376 MHz, $\left.\mathrm{CD}_{3} \mathrm{CN}\right): \sigma$ $-141.0\left(\mathrm{q}, 3 \mathrm{~F},{ }^{1} J_{\mathrm{BF}}=48.9 \mathrm{~Hz}, \mathrm{~B} F_{3}\right),-56.5\left(\mathrm{~s}, 3 \mathrm{~F}, \mathrm{OCF}_{3}\right) .{ }^{11} \mathrm{~B} \mathrm{NMR}\left(128 \mathrm{MHz}, \mathrm{CD}_{3} \mathrm{CN}\right): \sigma 2.99$ (q, ${ }^{1} J_{\mathrm{BF}}=50.8 \mathrm{~Hz}$ ). HRMS (FAB), $m / z$ : calcd. for $\mathrm{C}_{7} \mathrm{H}_{4} \mathrm{OBF}_{6} \mathrm{~K}_{2}{ }^{+} 306.9528[\mathrm{M}+\mathrm{K}]^{+}$; found 306.9535 . Elemental analysis calcd. for $\mathrm{C}_{7} \mathrm{H}_{4} \mathrm{OBF}_{6} \mathrm{~K}$ : $\mathrm{C}, 31.37 ; \mathrm{H}, 1.50$; found: $\mathrm{C}, 30.91 ; \mathrm{H}, 1.71$.

Potassium (ortho-methylthiophenyl)trifluoroborate $\left(\mathrm{K}\left[\boldsymbol{o}-\mathrm{SMeC}_{\mathbf{6}} \mathrm{H}_{\mathbf{4}} \mathrm{BF}_{3}\right]\right)$ : yield: $70 \%$, white solid. ${ }^{1} \mathrm{H}$ NMR (400 MHz, $\left.\mathrm{CD}_{3} \mathrm{CN}\right): \sigma 2.32\left(\mathrm{~s}, 3 \mathrm{H}, \mathrm{SCH}_{3}\right), 6.91(\mathrm{t}, 1 \mathrm{H}, J=7.6 \mathrm{~Hz}$, arom. H), $7.02-$ $7.10\left(\mathrm{~m}, 2 \mathrm{H}\right.$, arom. H), $7.40\left(\mathrm{~d}, 1 \mathrm{H},{ }^{3} J_{\mathrm{HH}}=6.9 \mathrm{~Hz}\right.$, arom. H). ${ }^{13} \mathrm{C}$ NMR $\left(100 \mathrm{MHz}, \mathrm{CD}_{3} \mathrm{CN}\right): \sigma 15.6$ (s, $\left.\mathrm{SCH}_{3}\right), 123.9$ (s), 124.1 (s), 127.5 (s), 133.1 (s), 142.6 (s). ${ }^{19} \mathrm{~F}$ NMR (376 MHz, $\left.\mathrm{CD}_{3} \mathrm{CN}\right): \sigma$ -140.4 (q, 3F, $\left.{ }^{1} J_{\mathrm{BF}}=52.4 \mathrm{~Hz}, \mathrm{~B} F_{3}\right) .{ }^{11} \mathrm{~B} \mathrm{NMR}\left(128 \mathrm{MHz}, \mathrm{CD}_{3} \mathrm{CN}\right): \sigma 3.37\left(\mathrm{q},{ }^{1} J_{\mathrm{BF}}=53.7 \mathrm{~Hz}\right)$. HRMS (FAB), $m / z$ : calcd. for $\mathrm{C}_{7} \mathrm{H}_{7} \mathrm{SBF}_{3} \mathrm{~K}_{2}{ }^{+} 268.9582[\mathrm{M}+\mathrm{K}]^{+}$; found 268.9586. Elemental analysis calcd. for $\mathrm{C}_{7} \mathrm{H}_{7} \mathrm{SBF}_{3} \mathrm{~K}: \mathrm{C}, 36.54 ; \mathrm{H}, 3.07$; found: $\mathrm{C}, 36.27 ; \mathrm{H}, 3.12$.

Potassium (ortho-methoxyphenyl)trifluoroborate $\left(\mathrm{K}\left[\boldsymbol{o}-\mathrm{OMeC}_{6} \mathrm{H}_{4} \mathrm{BF}_{3}\right]\right)$ : yield: $84 \%$, white solid. ${ }^{1} \mathrm{H}$ NMR (400 MHz, $\mathrm{CD}_{3} \mathrm{CN}$ ): $\sigma 3.68$ (s, 3H, OCH $), 6.74-6.77$ (m, 2H, arom. H), 7.08 (dt, 1H, $J=$ $7.8 \mathrm{~Hz},{ }^{4} J=1.8 \mathrm{~Hz}$, arom. H), $7.36\left(\mathrm{~d}, 1 \mathrm{H},{ }^{3} J_{\mathrm{HH}}=6.4 \mathrm{~Hz}\right.$, arom. $\left.\mathrm{H}\right) .{ }^{13} \mathrm{C} \mathrm{NMR} \mathrm{(100} \mathrm{MHz,}$ DMSO- $\left.d^{6}\right): \sigma 54.7\left(\mathrm{~s}, \mathrm{OCH}_{3}\right), 109.6(\mathrm{~s}), 119.1(\mathrm{~s}), 126.7$ (s), $133.2(\mathrm{~s}), 162.5(\mathrm{~s}) .{ }^{19} \mathrm{~F} \mathrm{NMR}(376 \mathrm{MHz}$, $\left.\mathrm{CD}_{3} \mathrm{CN}\right): \sigma-140.6\left(\mathrm{q}, 3 \mathrm{~F},{ }^{1} J_{\mathrm{BF}}=53.0 \mathrm{~Hz}, \mathrm{~B} F_{3}\right) .{ }^{11} \mathrm{~B} \mathrm{NMR}\left(128 \mathrm{MHz}, \mathrm{CD}_{3} \mathrm{CN}\right): \sigma 3.43\left(\mathrm{~d},{ }^{1} J_{\mathrm{BF}}=54.2\right.$ $\mathrm{Hz}$ ). HRMS (FAB), $m / z$ : calcd. for $\mathrm{C}_{7} \mathrm{H}_{7} \mathrm{OBF}_{3} \mathrm{~K}_{2}{ }^{+} 252.9811[\mathrm{M}+\mathrm{K}]^{+}$; found 252.9818. Elemental analysis calcd. for $\mathrm{C}_{7} \mathrm{H}_{7} \mathrm{OBF}_{3} \mathrm{~K}: \mathrm{C}, 39.28$; $\mathrm{H}, 3.30$; found: $\mathrm{C}, 39.15 ; \mathrm{H}, 3.32$.

Potassium (ortho-ethoxyphenyl)trifluoroborate $\left(\mathbf{K}\left[\boldsymbol{o}-\mathbf{O E t C}_{6} \mathbf{H}_{4} \mathbf{B F}_{3}\right]\right)$ : yield: $82 \%$, white solid. ${ }^{1} \mathrm{H}$ NMR (400 MHz, $\left.\mathrm{CD}_{3} \mathrm{CN}\right): \sigma 1.28\left(\mathrm{t}, 3 \mathrm{H},{ }^{3} \mathrm{~J}_{\mathrm{HH}}=7.1 \mathrm{~Hz}, \mathrm{OCH}_{2} \mathrm{CH}_{3}\right), 3.99\left(\mathrm{q}, 2 \mathrm{H},{ }^{3} J_{\mathrm{HH}}=7.1 \mathrm{~Hz}\right.$, $\left.\mathrm{OCH}_{2} \mathrm{CH}_{3}\right), 6.75-6.79(\mathrm{~m}, 2 \mathrm{H}$, arom. $\mathrm{H}), 7.08$ (dt, $1 \mathrm{H}, J=7.8 \mathrm{~Hz},{ }^{4} J=1.9 \mathrm{~Hz}$, arom. H), 7.38 (dd, $1 \mathrm{H}, J=7.2 \mathrm{~Hz},{ }^{4} J=1.2 \mathrm{~Hz}$, arom. H). ${ }^{13} \mathrm{C} \mathrm{NMR}\left(100 \mathrm{MHz}, \mathrm{CD}_{3} \mathrm{CN}\right): \sigma 15.3\left(\mathrm{~s}, \mathrm{OCH}_{2} \mathrm{CH}_{3}\right), 64.3(\mathrm{~s}$, $\mathrm{OCH}_{2} \mathrm{CH}_{3}$ ), 112.5 (s), 120.8 (s), 128.3 (s), 134.4 (s), 162.7 (s). ${ }^{19} \mathrm{~F}$ NMR (376 MHz, $\mathrm{CD}_{3} \mathrm{CN}$ ): $\sigma$ $-140.4\left(\mathrm{~d}, 3 \mathrm{~F},{ }^{1} J_{\mathrm{BF}}=58.9 \mathrm{~Hz}, \mathrm{~B} F_{3}\right) .{ }^{11} \mathrm{~B} \mathrm{NMR}\left(128 \mathrm{MHz}, \mathrm{CD}_{3} \mathrm{CN}\right): \sigma 3.67\left(\mathrm{~d},{ }^{1} J_{\mathrm{BF}}=48.8 \mathrm{~Hz}\right)$. HRMS (FAB), $m / z$ : calcd. for $\mathrm{C}_{8} \mathrm{H}_{9} \mathrm{OBF}_{3} \mathrm{~K}_{2}{ }^{+} 266.9967[\mathrm{M}+\mathrm{K}]^{+}$; found 266.9979. Elemental analysis calcd. for $\mathrm{C}_{8} \mathrm{H}_{9} \mathrm{OBF}_{3} \mathrm{~K}: \mathrm{C}, 42.13$; H, 3.98; found: $\mathrm{C}, 41.89$; H, 3.82 .

Potassium (ortho-nitrophenyl)trifluoroborate $\left(\mathbf{K}\left[\boldsymbol{o}-\mathrm{NO}_{2} \mathbf{C}_{6} \mathbf{H}_{4} \mathbf{B F}_{3}\right]\right)$ : yield: $82 \%$, gray solid. ${ }^{1} \mathrm{H}$ NMR (400 MHz, $\mathrm{CD}_{3} \mathrm{CN}$ ): $\sigma 7.27$ (dt, $1 \mathrm{H}, J=7.7 \mathrm{~Hz},{ }^{4} J=1.3 \mathrm{~Hz}$, arom. H), 7.39-7.45 (m, 2H, arom. H), 7.64 (d, $1 \mathrm{H},{ }^{3} J_{\mathrm{HH}}=7.3 \mathrm{~Hz}$, arom. H). $\left.{ }^{13} \mathrm{C} \mathrm{NMR} \mathrm{(100} \mathrm{MHz,} \mathrm{CD}_{3} \mathrm{CN}\right): \sigma 122.5$ (s), 127.9 (s), 
131.5 (s), 135.2 (s), 156.0 (s). ${ }^{19} \mathrm{~F}$ NMR (376 MHz, $\mathrm{CD}_{3} \mathrm{CN}$ ): $\sigma-141.8$ (q, 3F, ${ }^{1} J_{\mathrm{BF}}=46.3 \mathrm{~Hz}, \mathrm{~B} F_{3}$ ).

${ }^{11} \mathrm{~B}$ NMR (128 MHz, CD $\left.{ }_{3} \mathrm{CN}\right): \sigma 2.76\left(\mathrm{q},{ }^{1} J_{\mathrm{BF}}=48.4 \mathrm{~Hz}\right.$ ). HRMS (FAB), $m / z$ : calcd. for $\mathrm{C}_{6} \mathrm{H}_{4} \mathrm{NO}_{2} \mathrm{BF}_{3} \mathrm{~K}_{2}{ }^{+} 267.9556[\mathrm{M}+\mathrm{K}]^{+}$; found 267.9566. Elemental analysis calcd. for $\mathrm{C}_{6} \mathrm{H}_{4} \mathrm{NO}_{2} \mathrm{BF}_{3} \mathrm{~K}$ : C, 31.47; H, 1.76; N, 6.12; found: C, 31.47; H, 2.07; N, 6.11 .

Potassium (ortho-acetylphenyl)trifluoroborate $\left(\mathbf{K}\left[\boldsymbol{o}-\mathbf{C O C H}_{3} \mathbf{C}_{6} \mathbf{H}_{4} \mathbf{B F}_{3}\right]\right)$ : yield: $55 \%$, white solid. ${ }^{1} \mathrm{H}$ NMR (400 MHz, $\mathrm{CD}_{3} \mathrm{CN}$ ): $\sigma 2.45\left(\mathrm{~s}, 3 \mathrm{H}, \mathrm{COCH}_{3}\right), 7.13-7.15(\mathrm{~m}, 2 \mathrm{H}$, arom. H), 7.21-7.25 (m, $1 \mathrm{H}$, arom. H), $7.53\left(\mathrm{~d}, 1 \mathrm{H},{ }^{3} J_{\mathrm{HH}}=7.2 \mathrm{~Hz}\right.$, arom. H). ${ }^{13} \mathrm{C} \mathrm{NMR}\left(100 \mathrm{MHz}, \mathrm{CD}_{3} \mathrm{CN}\right): \sigma 31.3(\mathrm{~s}$, $\left.\mathrm{COCH}_{3}\right), 125.6$ (s), 126.5 (s), 129.3 (s), 133.7 (s), 146.9 (s), 210.2 (s, $\left.\mathrm{COCH}_{3}\right) .{ }^{19} \mathrm{~F} \mathrm{NMR}(376 \mathrm{MHz}$, $\left.\mathrm{CD}_{3} \mathrm{CN}\right): \sigma-138.5\left(\mathrm{q}, 3 \mathrm{~F},{ }^{1} J_{\mathrm{BF}}=51.0 \mathrm{~Hz}, \mathrm{~B} F_{3}\right) .{ }^{11} \mathrm{~B} \mathrm{NMR}\left(128 \mathrm{MHz}, \mathrm{CD}_{3} \mathrm{CN}\right): \sigma 3.44\left(\mathrm{q},{ }^{1} J_{\mathrm{BF}}=53.2\right.$ $\mathrm{Hz}$ ). HRMS (FAB), $m / z$ : calcd. for $\mathrm{C}_{8} \mathrm{H}_{7} \mathrm{OBF}_{3} \mathrm{~K}_{2}{ }^{+} 264.9811[\mathrm{M}+\mathrm{K}]^{+}$; found 264.9819. Elemental analysis calcd. for $\mathrm{C}_{8} \mathrm{H}_{7} \mathrm{OBF}_{3} \mathrm{~K}: \mathrm{C}, 42.51 ; \mathrm{H}, 3.12$; found: $\mathrm{C}, 42.60 ; \mathrm{H}, 3.18$.

Potassium (2-fluoro-5-methoxyphenyl)trifluoroborate $\left(\mathrm{K}\left[2-\mathrm{F}-5-\mathrm{OMeC}_{6} \mathbf{H}_{3} \mathrm{BF}_{3}\right]\right)$ : yield: $90 \%$, white solid. ${ }^{1} \mathrm{H}$ NMR (400 MHz, $\mathrm{CD}_{3} \mathrm{CN}$ ): $\sigma 3.96$ (s, 3H, $\left.\mathrm{OCH}_{3}\right), 6.60-6.65(\mathrm{~m}, 1 \mathrm{H}$, arom. H), 6.75 $\left(\mathrm{t}, 1 \mathrm{H}, J=8.7 \mathrm{~Hz}\right.$, arom. H), 6.93-6.95 (m, 1H, arom. H). $\left.{ }^{13} \mathrm{C} \mathrm{NMR} \mathrm{(100} \mathrm{MHz,} \mathrm{CD}_{3} \mathrm{CN}\right): \sigma 56.0(\mathrm{~s}$, $\left.\mathrm{OCH}_{3}\right), 113.5\left(\mathrm{~d},{ }^{3} J_{\mathrm{CF}}=9.6 \mathrm{~Hz}\right), 115.3\left(\mathrm{~d},{ }^{2} J_{\mathrm{CF}}=29.7 \mathrm{~Hz}\right), 119.3\left(\mathrm{~d},{ }^{3} J_{\mathrm{CF}}=12.9 \mathrm{~Hz}\right), 156.1(\mathrm{~s}), 161.3$ $\left(\mathrm{d},{ }^{1} J_{\mathrm{CF}}=229.9 \mathrm{~Hz}\right) .{ }^{19} \mathrm{~F} \mathrm{NMR}\left(376 \mathrm{MHz}, \mathrm{CD}_{3} \mathrm{CN}\right): \sigma-141.0\left(\mathrm{~d}, 3 \mathrm{~F},{ }^{1} J_{\mathrm{BF}}=53.2 \mathrm{~Hz}, \mathrm{~B} F_{3}\right),-122.7(\mathrm{~s}$, $1 \mathrm{~F}, 2-F) .{ }^{11} \mathrm{~B}$ NMR $\left(128 \mathrm{MHz}, \mathrm{CD}_{3} \mathrm{CN}\right): \sigma 3.06\left(\mathrm{~d},{ }^{1} J_{\mathrm{BF}}=48.8 \mathrm{~Hz}\right)$. HRMS (FAB), $m / z$ : calcd. for $\mathrm{C}_{7} \mathrm{H}_{6} \mathrm{OBF}_{4} \mathrm{~K}_{2}{ }^{+} 270.9716[\mathrm{M}+\mathrm{K}]^{+}$; found 270.9727. Elemental analysis calcd. for $\mathrm{C}_{7} \mathrm{H}_{6} \mathrm{OBF}_{4} \mathrm{~K}: \mathrm{C}$, 36.24; H, 2.61; found: C, 36.08; H, 2.82.

Potassium (2-chloro-5-trifluoromethylphenyl)trifluoroborate (K[2-Cl-5- $\mathbf{C F}_{\mathbf{3}} \mathbf{C}_{\mathbf{6}} \mathbf{H}_{\mathbf{3}} \mathbf{B F}$ ]): yield: $85 \%$, white solid. ${ }^{1} \mathrm{H}$ NMR (400 MHz, DMSO- $\left.d^{6}\right): \sigma 7.36\left(\mathrm{~d}, 1 \mathrm{H},{ }^{3} J_{\mathrm{HH}}=8.2 \mathrm{~Hz}\right.$, arom. H), 7.42 (dd, $1 \mathrm{H},{ }^{3} J_{\mathrm{HH}}=8.2 \mathrm{~Hz},{ }^{4} \mathrm{~J}=2.3 \mathrm{~Hz}$, arom. H), 7.70 (s, 1H, arom. H). ${ }^{13} \mathrm{C}$ NMR (100 MHz, DMSO- $\left.d^{6}\right): \sigma$ $124.0\left(\mathrm{q},{ }^{3} J_{\mathrm{CF}}=3.3 \mathrm{~Hz}\right), 124.8\left(\mathrm{q},{ }^{1} J_{\mathrm{CF}}=270.5 \mathrm{~Hz}, C \mathrm{~F}_{3}\right), 125.8\left(\mathrm{q},{ }^{2} J_{\mathrm{CF}}=30.3 \mathrm{~Hz}\right), 129.0(\mathrm{~s}), 130.1$ $\left(\mathrm{q},{ }^{3} J_{\mathrm{CF}}=3.1 \mathrm{~Hz}\right), 141.9(\mathrm{~s}) .{ }^{19} \mathrm{~F}$ NMR $\left(376 \mathrm{MHz}, \mathrm{DMSO}-d^{6}\right): \sigma-142.2\left(\mathrm{br}, 3 \mathrm{~F}, \mathrm{~B} F_{3}\right),-63.3$ (s, 3F, $\left.\mathrm{C} F_{3}\right) .{ }^{11} \mathrm{~B}$ NMR $\left(128 \mathrm{MHz}, \mathrm{DMSO}-d^{6}\right): \sigma 2.59\left(\mathrm{~d},{ }^{1} J_{\mathrm{BF}}=45.0 \mathrm{~Hz}\right)$. HRMS (FAB), $m / z$ : calcd. for $\mathrm{C}_{7} \mathrm{H}_{3} \mathrm{ClBF}_{6} \mathrm{~K}_{2}{ }^{+} 324.9189[\mathrm{M}+\mathrm{K}]^{+}$; found 324.9191. Elemental analysis calcd. for $\mathrm{C}_{7} \mathrm{H}_{3} \mathrm{ClBF}_{6} \mathrm{~K}: \mathrm{C}$, 29.35; H, 1.06; found: C, 29.80; H, 1.26 .

Potassium (3-methoxycarbonyl-5-nitrophenyl)trifluoroborate $\left(\mathrm{K}\left[3-\mathrm{CO}_{\mathbf{2}} \mathrm{Me}-5-\mathrm{NO}_{\mathbf{2}} \mathrm{C}_{\mathbf{6}} \mathrm{H}_{\mathbf{3}} \mathrm{BF}_{\mathbf{3}}\right]\right.$ ): yield: 73\%, white solid. ${ }^{1} \mathrm{H}$ NMR (400 MHz, DMSO- $d^{6}$ ): $\sigma 3.91\left(\mathrm{~s}, 3 \mathrm{H}, \mathrm{CO}_{2} \mathrm{CH}_{3}\right.$ ), 8.35 (br, $2 \mathrm{H}$, arom. $\mathrm{H}), 8.44$ (s, 1H, arom. H). ${ }^{13} \mathrm{C}$ NMR (100 MHz, DMSO- $\left.d^{6}\right): \sigma 52.5\left(\mathrm{~s}, \mathrm{CO}_{2} \mathrm{CH}_{3}\right), 121.0(\mathrm{~s}), 129.4(\mathrm{~s})$, 129.5 (s), 138.1 (s), 147.1 (s), $165.6\left(\mathrm{~s}, \mathrm{CO}_{2} \mathrm{CH}_{3}\right) .{ }^{19} \mathrm{~F}$ NMR (376 MHz, DMSO- $d^{6}$ ): $\sigma-143.3$ (br, 3F, $\left.\mathrm{B} F_{3}\right) .{ }^{11} \mathrm{~B}$ NMR $\left(128 \mathrm{MHz}, \mathrm{DMSO}-d^{6}\right): \sigma 2.87$ (br). HRMS (FAB), $m / z$ : calcd. for $\mathrm{C}_{8} \mathrm{H}_{6} \mathrm{NO}_{4} \mathrm{BF}_{3} \mathrm{~K}_{2}{ }^{+}$ 325.9611 $[\mathrm{M}+\mathrm{K}]^{+}$; found 325.9612. Elemental analysis calcd. for $\mathrm{C}_{8} \mathrm{H}_{6} \mathrm{NO}_{4} \mathrm{BF}_{3} \mathrm{~K}: \mathrm{C}, 33.48 ; \mathrm{H}$, 2.11; N, 4.88; found: $\mathrm{C}, 33.32 ; \mathrm{H}, 2.36 ; \mathrm{N}, 6.03$. 
Potassium 3-thienyltrifluoroborate $\left(\mathbf{K}\left[\mathbf{3}-\mathbf{C}_{\mathbf{4}} \mathbf{H}_{\mathbf{3}} \mathbf{S B B} \mathbf{F}_{\mathbf{3}}\right]\right)$ : yield: $97 \%$, gray solid ${ }^{1} \mathrm{H}$ NMR (400 MHz, DMSO- $\left.d^{6}\right): \sigma 6.97-7.00\left(\mathrm{~m}, 2 \mathrm{H}\right.$, arom. H), 7.16-7.18 (m, 1H, arom. H). ${ }^{13} \mathrm{C}$ NMR (100 MHz, DMSO- $\left.d^{6}\right): \sigma 122.5(\mathrm{~s}), 124.2(\mathrm{~s}), 131.9(\mathrm{~s}) .{ }^{19} \mathrm{~F}$ NMR $\left(376 \mathrm{MHz}, \mathrm{DMSO}-d^{6}\right): \sigma-138.0\left(\mathrm{q}, 3 \mathrm{~F},{ }^{1} J_{\mathrm{BF}}=\right.$ $\left.52.0 \mathrm{~Hz}, \mathrm{~B} F_{3}\right) .{ }^{11} \mathrm{~B}$ NMR $\left(128 \mathrm{MHz}, \mathrm{DMSO}-d^{6}\right): \sigma 2.96$ (q, $\left.{ }^{1} J_{\mathrm{BF}}=50.8 \mathrm{~Hz}\right)$. HRMS (FAB), $m / z$ : calcd. for $\mathrm{C}_{4} \mathrm{H}_{3} \mathrm{SBF}_{3} \mathrm{~K}_{2}{ }^{+} 228.9269[\mathrm{M}+\mathrm{K}]^{+}$; found 228.9282. Elemental analysis calcd. for $\mathrm{C}_{4} \mathrm{H}_{3} \mathrm{SBF}_{3} \mathrm{~K}: \mathrm{C}$, 25.28; H, 1.59; found: C, 25.08; H, 1.85 .

Potassium 1-naphthyltrifluoroborate $\left(\mathbf{K}\left[1-\mathbf{N a p h B F}_{3}\right]\right)$ : yield: $81 \%$, pale brown solid ${ }^{1} \mathrm{H}$ NMR $\left(400 \mathrm{MHz}, \mathrm{DMSO}-d^{6}\right): \sigma 7.26-7.32(\mathrm{~m}, 3 \mathrm{H}$, arom. H), 7.55-7.59 (m, 2H, arom. H), 7.70-7.72 (m, $1 \mathrm{H}$, arom. H), 8.39 (d, $\left.1 \mathrm{H},{ }^{3} J_{\mathrm{HH}}=8.7 \mathrm{~Hz}\right) .{ }^{13} \mathrm{C} \mathrm{NMR}\left(100 \mathrm{MHz}, \mathrm{DMSO}-d^{6}\right): \sigma 123.5(\mathrm{~s}), 124.0(\mathrm{~s})$, $125.0(\mathrm{~s}), 125.3$ (s), 127.5 (s), 128.6 (d, $\left.J_{\mathrm{CF}}=2.9 \mathrm{~Hz}\right), 130.3$ (s), 133.0 (s), 136.6 (s). ${ }^{19} \mathrm{~F}$ NMR (376 MHz, DMSO- $\left.d^{6}\right): \sigma-137.7\left(\mathrm{~d}, 3 \mathrm{~F},{ }^{1} J_{\mathrm{BF}}=44.8 \mathrm{~Hz}, \mathrm{~B} F_{3}\right) .{ }^{11} \mathrm{~B}$ NMR $\left(128 \mathrm{MHz}, \mathrm{DMSO}-d^{6}\right): \sigma 4.12(\mathrm{~d}$, ${ }^{1} J_{\mathrm{BF}}=46.9 \mathrm{~Hz}$ ). HRMS (FAB), $m / z$ : calcd. for $\mathrm{C}_{10} \mathrm{H}_{7} \mathrm{BF}_{3} \mathrm{~K}_{2}^{+} 272.9862[\mathrm{M}+\mathrm{K}]^{+}$; found 272.9868 . Elemental analysis calcd. for $\mathrm{C}_{10} \mathrm{H}_{7} \mathrm{BF}_{3} \mathrm{~K}$ : C, 51.31; H, 3.01; found: C, 50.56; H, 2.90.

The signal of ${ }^{13} \mathrm{C}$ binding ${ }^{11} \mathrm{~B}$ atom was too broad due to the quadrupolar relaxation mechanism of ${ }^{11}$ B nucleus. ${ }^{1}$

\section{REFERENCE}

(1) Oliveira, R. A.; Silva, R. O.; Molander, G. A.; Menezes, P. H. ${ }^{1} \mathrm{H},{ }^{13} \mathrm{C},{ }^{19} \mathrm{~F}$ and ${ }^{11} \mathrm{~B}$ NMR spectral reference data of some potassium organotrifluoroborates. Magn. Reson. Chem. 2009, 47, 873-878. 